\title{
Effects of Varying CDS Levels and Drying and Cooling Temperatures on Flowability Properties of DDGS
}

\author{
Rumela Bhadra, ${ }^{1}$ Kasiviswanathan Muthukumarappan, ${ }^{2,3}$ and Kurt A. Rosentrater ${ }^{4}$
}

ABSTRACT

Cereal Chem. 90(1):35-46

\begin{abstract}
Demand for alternative fuels and the need to reduce dependence on fossil fuels have triggered the growth of corn-based ethanol production, which is expected to rise in future years. Transportation of the coproduct distillers dried grains with solubles (DDGS) from this industry occurs under various environmental conditions. Transporting DDGS is often problematic, because caking between the particles can lead to flow problems. In this study, we have prepared DDGS by combining condensed distillers solubles (CDS) with distillers wet grains and then drying. We investigated the effects of CDS level (10, 15, and 20\%, wb), drying temperature (100, 200, and $\left.300^{\circ} \mathrm{C}\right)$, and cooling temperature $\left(-12,25\right.$, and $\left.35^{\circ} \mathrm{C}\right)$ on the flowability of the resulting DDGS. Statistical analyses of the resulting data found signifi-
\end{abstract}

cant differences among the cooling temperature levels for angle of repose, total flow and flood indices, dispersibility, water activity, and protein dispersibility index. Additionally, significant interaction effects between CDS, drying temperature, and cooling temperature levels for angle of repose, total flow and flood indices, dispersibility, and protein dispersibility index were observed. Response surface regression on selected dimensionless flowability parameters was also applied. However, multivariate PLS regression yielded better results $\left(R^{2}>0.8\right)$ than response surface plots. Understanding the effects of drying and cooling temperatures as well as CDS levels can be used to help improve the industrial processing of DDGS and improve storage and transportation.
The final quality of powders or particulates can depend on their composition and consistent initial mixing and handling. Powder composition, in turn, can depend on material physical properties such as particle size and shape, particle density, humidity, and environmental factors such as moisture, temperature, and time (Abu-hardan and Hill 2010). Bulk properties of particulate substances are important because physical and environmental properties affect particulate substance behavior during handling, storage, transportation, mixing, compression, packing, and processing (Knowlton et al 1994; Fitzpatrick et al 2004). With the substantial growth of the U.S. corn-based fuel ethanol industry, a large amount of corn-based distillers dried grains with solubles (DDGS) is being produced. The production of DDGS in the crop year 2010-2011 was calculated as 34.1 million metric tons (0.9 million metric tons from beverage ethanol and 33.2 million metric tons from fuel ethanol), and production is projected to grow (Renewable Fuels Association 2012). DDGS has been growing in importance as livestock feed for cattle, swine, and in some cases poultry for over two decades because of its high energy content and moderate protein values $(28-34 \%, \mathrm{db})$ and trace amounts of essential amino acids like methionine, leucine, arginine, and threonine (Speihs et al 2002; Rosentrater and Muthukumarappan 2006). To meet the high demand for DDGS in the livestock feed industry, transporting and handling DDGS over long distances (generally by rail) is essential. Thus, DDGS can be exposed to different environmental and physical conditions such as temperature fluctuations, moisture migrations, and humidity changes.

\footnotetext{
*The $\boldsymbol{e}$-Xtra logo stands for "electronic extra" and indicates that Figures 4-9 appear in color online.

${ }^{1}$ Department of Biological and Agricultural Engineering, Kansas State University, Manhattan, KS 66506-2906, U.S.A. Mention of trade name, propriety product or specific equipment does not constitute a guarantee or warranty by the United States Department of Agriculture and does not imply approval of a product to the exclusion of others that may be suitable.

${ }^{2}$ Department of Agricultural and Biosystems Engineering, South Dakota State University, Agricultural Engineering, Brookings, SD 57007, U.S.A.

${ }^{3}$ Corresponding author. Phone: (605) 688-5141. Fax: (605) 688-6764. E-mail: kas.muthukum@sdstate.edu

${ }^{4}$ Department of Agricultural and Biosystems Engineering, Iowa State University, 3167 NSRIC Building, Ames, IA 50011, U.S.A.
}

http://dx.doi.org/10.1094/CCHEM-04-12-0042-R

(C) 2013 AACC International, Inc.
Like many agricultural and food materials, DDGS is hygroscopic in nature (i.e., it has the ability to absorb moisture when exposed to humid conditions during the handling and storage of the material). Because of temperature differences, fat content, and prolonged consolidation in storage, DDGS particles may tend to agglomerate to form solidified cakes. Caking, or hardening, of DDGS leads to difficulties during its unloading from rail cars and storage vessels; it significantly contributes to economic loss and involves labor to break up the agglomerates or cakes (Rock and Schwedes 2005). Caking of biomaterials takes place because of interparticle attraction and is regulated by the strength of the attractive and gravitational forces. For particles in an amorphous state, interparticle forces, liquid bridges, and solid bridges are the primary causes for particle caking (Barbosa-Canovas and Juliano 2005).

Moisture also plays a significant role in caking and flowability of particulate material. Abu-hardan and Hill (2010) found that for wheat cereals with water content higher than $30 \%$, it was not possible to measure stickiness, and hence, higher cohesion indices with an increase in water content were observed. DDGS also showed flowability problems with an increase in moisture content. According to Bhadra et al (2009d), $T_{\mathrm{s}}$ (sticky point temperature) decreased when the moisture content increased, indicating more stickiness and flow problems in DDGS.

Storage temperature can also affect the flowability of particulate substances. Whole milk particles showed changes in flowability resulting from differences in storage temperatures. Jenike (1964) flow index $(-)$ showed lower values (indices $<4$ ), indicating higher cohesiveness, in milk particulates resulting from increase in the storage temperature from 15 to $25^{\circ} \mathrm{C}$ (Fitzpatrick et al 2004). Increased cohesion was likely because of partial melting of milk fat resulting in the formation of liquid bridges between particles. Furthermore, during storage at cooler ambient temperatures, the fat molecules underwent solidification and formed solid bridges between particles. These conditions can greatly impact particle cohesiveness and may lead to potential flow problems (Bhadra et al 2010).

If particulates are exposed to compressive stress for some period of time, particles may gain strength and develop flow problems (Jenike 1964). Teunou and Fitzpatrick (2000) indicated that a scientific way to account for the effect of particulate material flow properties as a function of storage time was through Jenike (1964) flow functions of time consolidation. Such flow function 
curves measured by the Jenike shear cell are called temporal flow function curves. For tea and whey permeate powders and particulates, they found that temporal flow function curves showed more flowability problems when the consolidation time was increased up to one week. To investigate the flow problems of bulk solids, it is necessary to understand the material properties that may affect flowability. The flow properties are dependent on chemical composition, particle size distribution, product temperature, moisture content, thermal properties, and compacting pressure. Except pressure, all of these properties are inherent material characteristics of a bulk solid. Pressure developed depends on size of the bin, resulting in scale-up problems.

Many testers and methods have been studied to measure strength and flow properties of bulk solids (Geldart et al 2009; Abu-hardan and Hill 2010). The most important parameters to measure flowability in particulate materials include effective angle of internal friction $\left(\delta,{ }^{\circ}\right)$, angle of internal friction $\left(\phi,{ }^{\circ}\right)$, unconfined yield strength $\left(\sigma_{c}, \mathrm{~Pa}\right)$, compressibility factor $(-)$, and bulk density $\left(\mathrm{kg} / \mathrm{m}^{3}\right)$ (Johanson 1972).

Along these lines, Jenike (1964) developed a test procedure to measure flow properties. Jenike flow function is obtained from the plot of the unconfined yield strength versus major consolidation stress, and it represents the strength developed within a bulk material. This strength in particulate substances must be overcome to make it flow. A flow function curve lying toward the $x$ axis of the plot represents "easy flow," but it becomes "difficult flow" as it moves upward in an anticlockwise direction (Fig. 1). Jenike (1964) flow index, which is defined as the ratio of major consolidation stress to the unconfined yield strength, is also the inverse slope of the flow function. Flow index helps to categorize the bulk materials and can indicate the propensity for caking problems in a particulate substance. More details on the minimum width of the hopper outlet, mass flow, flow factor, and overall flowability can be found in Jenike (1964) and Johanson (1972).

According to Kamath et al (1994), the Jenike shear cell method is chosen in preference to triaxial methods because of several advantages, and it is thus a very widely used flow property measuring device in industry. Apart from flow function indices, the slope of a yield-locus-obtained Mohr circle is also used to evaluate flowability and cohesion in wheat flour. The slope of the plot of shear stress $(T)$ versus normal stress $(\Phi)$ gives the Jenike (1964) yield loci for particulates (Kamath et al 1994).

Test procedures developed by Carr (1965) were also used to evaluate the flowability of different types of DDGS. DDGS obtained from commercial plants and reduced-fat DDGS were subjected to Carr flow property and Jenike shear analysis. More details can be found in Bhadra et al (2009a, 2009c) and Ganesan et

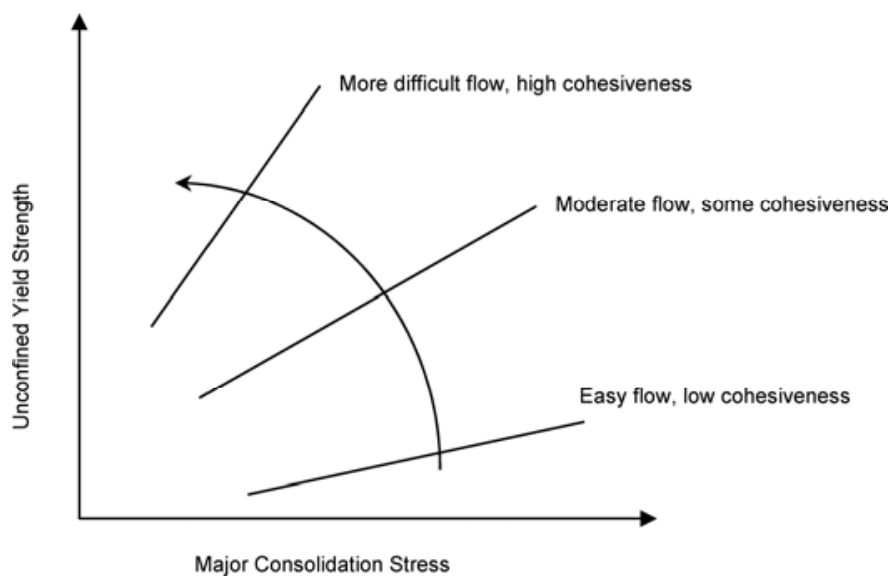

Fig. 1. Generalized Jenike flow functions indicating easy and difficult flow, based on Fitzpatrick et al (2004). al (2007b, 2008). DDGS produced with varying soluble and moisture contents was also examined (Ganesan et al 2007a). Apart from typical physical and flow property studies on DDGS, reports are also available on glass transition temperature $\left(T_{\mathrm{g}}\right)$ and sticky point temperature $\left(T_{\mathrm{s}}\right)$ behavior of DDGS with varying condensed distillers solubles (CDS), drying temperature, and storage temperature levels (Bhadra et al 2009d, 2010). These studies provide important information on flowability of DDGS and can be used to prevent particle stickiness and caking in industrial scenarios.

CDS is often referred to as "syrup" in the industry. CDS, another byproduct generated from corn-based bioethanol plants, is mixed with wet distillers cake and is subsequently dried to form DDGS. CDS is high in vitamins, fat, and protein but low in fiber content, and it yields a digestible energy value of approximately 91\% that of raw corn (Buchheit 2002; Cruz et al 2005).

Until now, only one study has been performed to examine flowability properties with drying temperatures and CDS levels, but all the laboratory-prepared DDGS samples were cooled at $25^{\circ} \mathrm{C}$ (Bhadra et al 2009c). Information about the flowability behavior of DDGS with varying drying temperature, cooling temperature, and CDS levels was not yet available. Specific objectives of this study were 1) to prepare DDGS with varying CDS levels $(10,15$, and $20 \%$, wb) and drying temperature levels (100, 200, and $\left.300^{\circ} \mathrm{C}\right)$; 2) to cool the DDGS samples at varying cooling temperature levels $\left(-12,25\right.$, and $\left.35^{\circ} \mathrm{C}\right)$ and to study the effect of cooling temperatures on DDGS handling; 3 ) to measure Carr and Jenike flow properties of the resulting DDGS; and 4) to develop a comprehensive overall regression model for flow parameters, evaluating the effects of varying CDS, drying temperature, and cooling temperature levels. An in-depth analysis of factors affecting flowability of DDGS will provide an understanding about storage and handling effects under varying processing and environmental conditions.

\section{MATERIALS AND METHODS}

\section{Sample Collection}

Samples of distillers wet grains (DWG) and CDS were collected from a commercial fuel ethanol plant in South Dakota and were stored under cold conditions $\left(-10 \pm 1^{\circ} \mathrm{C}\right)$.

\section{Sample Preparation}

CDS was added to the DWG at levels of 10,15 , and $20 \%$ (wb) and then mixed thoroughly (mixer model no. D300, Hobart Corporation, Troy, OH, U.S.A.) for $5 \mathrm{~min}$ for each CDS addition level. Then, $300 \mathrm{~g}$ of each mixture was spread uniformly onto a thin steel tray with the dimensions of $38 \times 27 \times 1 \mathrm{~cm}$, and it was dried in a laboratory scale oven $(838$ F, Fisher Scientific, Pittsburg, PA, U.S.A.). The drying was done at three selected temperatures of 100,200 , and $300^{\circ} \mathrm{C}$; temperature selection was based on interviews and discussions with industry experts, as well as on our studies of DDGS drying rate and moisture content (Bhadra et al 2009c).

For each temperature and CDS combination, the drying operation was done for specific times to reduce all the experimental samples to $8 \%(\mathrm{db})$ moisture content, which was the common baseline moisture content, thus eliminating the possible influence on the flowability parameters. After drying, each sample was cooled and stored in the refrigerator at $-12^{\circ} \mathrm{C}$ and in an oven at $35^{\circ} \mathrm{C}$. For both the cases, the flow and physical property measurements were carried out within a month of preparation. Thus, in total we had 18 ( 3 drying temperatures $\times 2$ cooling temperatures $\times 3$ CDS levels) experimental runs for all the property measurements.

The flow property data obtained from our previous study (Bhadra et al 2009c) with similar drying temperatures and CDS levels but cooled at room temperature $\left(25^{\circ} \mathrm{C}\right)$ were pooled with the data obtained in this study. 


\section{Experimental Design}

This study was conducted with a $3 \times 3 \times 3=27$ full factorial design for three drying temperature $\left(100,200\right.$, and $\left.300^{\circ} \mathrm{C}\right)$, three cooling temperature $\left(-12,25\right.$, and $\left.35^{\circ} \mathrm{C}\right)$, and three CDS addition $(10,15$, and $20 \%$, wb) levels. These treatment combinations (27 treatments in total) were implemented following a completely randomized design, and all measurements on the DDGS samples were completed four times, for each property.

\section{Physical Property Measurements}

Thermal properties (conductivity, diffusivity, and resistivity) were determined with a thermal properties meter (KD2, Decagon Devices, Pullman, WA, U.S.A.) that utilized the line heat source probe technique. Geometric mean diameter was determined following ASAE 34 standard method S319.3 via particle size distribution by Rotap sieve analyzer (model RX-29, Tyler Manufacturing, Mentor, OH, U.S.A.). Color was measured with a spectrocolorimeter (LabScan XE, Hunter Associates Laboratory, Reston, VA, U.S.A.) and the $L-a-b$ opposable color scales. Water activity was measured with a calibrated water activity meter (AW Sprint TH 500, Novasina, Talstrasse, Switzerland).
Porosity of the DDGS samples was calculated from the method described in Sahin and Sumnu (2006) and Chang (1988) using a multivolume pycnometer (Micromeritics, Norcross, GA, U.S.A.). The protein dispersibility index (PDI) was calculated following AACC International Approved Method 46-24.01 for determining protein dispersibility for full-fat, defatted, and whole or ground soy flour or soybeans.

\section{Flow Property Measurements}

Carr index tests were used to measure the flow properties of the DDGS samples. A powder characteristics tester (model PTR, Hosokawa Micron Powder Systems, Summit, NJ, U.S.A.) was used to measure the Carr (1965) flow properties, following the procedure described by ASTM (1999). The Carr flow properties included angle of repose (AoR), aerated bulk density (ABD), packed bulk density (PBD), Carr compressibility $\left(C_{\mathrm{c}}\right)$, uniformity, angle of fall (AoF), angle of spatula (AoS), angle of difference (AoD), and dispersibility. These parameters were then used to determine both the total flowability index (sum of AoR, $C_{\mathrm{c}}$, AoS, and uniformity) and total floodability index (sum of flowability index, AoD, AoF, and dispersibility). Hausner ratio (HR) is de-

TABLE I

Main Effects Due to Drying Temperature, Cooling Temperature, and CDS Level on Flow and Physical Properties of DDGS

\begin{tabular}{|c|c|c|c|c|c|c|c|c|c|}
\hline \multirow[b]{2}{*}{ Properties } & \multicolumn{3}{|c|}{ Drying Temperature $\left({ }^{\circ} \mathrm{C}\right)$} & \multicolumn{3}{|c|}{ Cooling Temperature $\left({ }^{\circ} \mathrm{C}\right)$} & \multicolumn{3}{|c|}{$\operatorname{CDS}(\%, w b)$} \\
\hline & 100 & 200 & 300 & -12 & 25 & 35 & 10 & 15 & 20 \\
\hline Angle of repose $\left(^{\circ}\right)$ & $\begin{array}{c}42.37 \mathrm{~b} \\
(1.64)\end{array}$ & $\begin{array}{c}45.46 \mathrm{a} \\
(1.41)\end{array}$ & $\begin{array}{c}45.96 a \\
(1.29)\end{array}$ & $\begin{array}{c}44.63 \mathrm{a} \\
(2.07)\end{array}$ & $\begin{array}{c}44.50 b \\
(1.56)\end{array}$ & $\begin{array}{c}44.57 \mathrm{~b} \\
(2.24)\end{array}$ & $\begin{array}{c}44.45 b \\
(1.98)\end{array}$ & $\begin{array}{c}43.45 b \\
(2.52)\end{array}$ & $\begin{array}{c}45.43 \mathrm{a} \\
(1.63)\end{array}$ \\
\hline Aerated bulk density $\left(\mathrm{kg} / \mathrm{m}^{3}\right)$ & $\begin{array}{r}444.00 \mathrm{~b} \\
(0.02)\end{array}$ & $\begin{array}{r}450.00 \mathrm{~b} \\
(0.02)\end{array}$ & $\begin{array}{r}460.56 \mathrm{a} \\
(0.02)\end{array}$ & $\begin{array}{c}\mathbf{4 4 4 . 0 0 a} \\
(0.05)\end{array}$ & $\begin{array}{r}\text { 450.01a } \\
(0.75)\end{array}$ & $\begin{array}{c}\text { 440.00a } \\
(0.84)\end{array}$ & $\begin{array}{c}445.71 \mathrm{a} \\
(0.05)\end{array}$ & $\begin{array}{c}445.85 \mathrm{ab} \\
(0.02)\end{array}$ & $\begin{array}{r}444.05 \mathrm{~b} \\
(0.03)\end{array}$ \\
\hline Particle size $\left(d_{\mathrm{gw}}, \mathrm{mm}\right)$ & $\begin{array}{c}0.86 \mathrm{a} \\
(0.27)\end{array}$ & $\begin{array}{c}0.75 b \\
(0.08)\end{array}$ & $\begin{array}{c}0.86 \mathrm{a} \\
(0.19)\end{array}$ & $\begin{array}{c}\mathbf{0 . 8 4 a} \\
(0.12)\end{array}$ & $\begin{array}{c}\mathbf{0 . 8 2 a} \\
(0.11)\end{array}$ & $\begin{array}{c}\text { 0.80a } \\
(0.15)\end{array}$ & $\begin{array}{c}0.70 \mathrm{c} \\
(0.06)\end{array}$ & $\begin{array}{c}0.76 b \\
(0.07)\end{array}$ & $\begin{array}{c}1.02 \mathrm{a} \\
(0.18)\end{array}$ \\
\hline Hausner ratio $(-)$ & $\begin{array}{c}1.12 \mathrm{a} \\
(0.11)\end{array}$ & $\begin{array}{c}1.13 \mathrm{~b} \\
(0.05)\end{array}$ & $\begin{array}{c}1.10 \mathrm{c} \\
(0.05)\end{array}$ & $\begin{array}{c}1.18 \mathrm{a} \\
(0.03)\end{array}$ & $\begin{array}{c}1.14 b \\
(0.25)\end{array}$ & $\begin{array}{l}1.11 b \\
(0.11)\end{array}$ & $\begin{array}{c}1.16 \mathrm{a} \\
(1.96)\end{array}$ & $\begin{array}{l}1.13 b \\
(2.53)\end{array}$ & $\begin{array}{r}1.14 \mathrm{a} \\
(1.63)\end{array}$ \\
\hline Total flow index (-) & $\begin{array}{c}76.13 a \\
(2.63)\end{array}$ & $\begin{array}{c}76.22 \mathrm{a} \\
(1.82)\end{array}$ & $\begin{array}{c}74.81 b \\
(2.85)\end{array}$ & $\begin{array}{c}75.48 \mathrm{a} \\
(2.17)\end{array}$ & $\begin{array}{c}76.49 \mathrm{a} \\
(1.52)\end{array}$ & $\begin{array}{c}76.96 b \\
(2.98)\end{array}$ & $\begin{array}{c}75.60 \mathrm{a} \\
(2.21)\end{array}$ & $\begin{array}{c}75.6 \mathrm{ab} \\
(2.51)\end{array}$ & $\begin{array}{l}75.29 \mathrm{~b} \\
(2.45)\end{array}$ \\
\hline Jenike flow function index (-) & $\begin{array}{c}\text { 3.21a } \\
(3.21)\end{array}$ & $\begin{array}{c}\mathbf{3 . 2 8 a} \\
(3.28)\end{array}$ & $\begin{array}{c}\text { 3.37a } \\
(3.37)\end{array}$ & $\begin{array}{c}2.18 \mathrm{c} \\
(0.94)\end{array}$ & $\begin{array}{c}5.21 \mathrm{a} \\
(0.78)\end{array}$ & $\begin{array}{c}3.77 \mathrm{~b} \\
(1.05)\end{array}$ & $\begin{array}{c}\text { 3.38a } \\
(1.33)\end{array}$ & $\begin{array}{c}\mathbf{3 . 2 0 a} \\
(1.05)\end{array}$ & $\begin{array}{c}\text { 3.38a } \\
(1.62)\end{array}$ \\
\hline Protein dispersibility index (\%) & $\begin{array}{c}9.19 \mathrm{a} \\
(0.49)\end{array}$ & $\begin{array}{c}7.45 b \\
(0.79)\end{array}$ & $\begin{array}{c}5.99 \mathrm{c} \\
(0.76)\end{array}$ & $\begin{array}{c}7.73 \mathrm{a} \\
(1.32)\end{array}$ & $\begin{array}{c}8.03 \mathrm{a} \\
(1.57)\end{array}$ & $\begin{array}{c}7.36 \mathrm{~b} \\
(1.63)\end{array}$ & $\begin{array}{c}6.12 \mathrm{a} \\
(1.47)\end{array}$ & $\begin{array}{c}7.37 \mathrm{~b} \\
(1.43)\end{array}$ & $\begin{array}{l}7.14 \mathrm{~b} \\
(1.38)\end{array}$ \\
\hline Compressibility (\%) & $\begin{array}{c}14.07 \mathrm{a} \\
(4.37)\end{array}$ & $\begin{array}{l}11.26 \mathrm{~b} \\
(3.51)\end{array}$ & $\begin{array}{l}11.31 b \\
(3.32)\end{array}$ & $\begin{array}{c}14.01 \mathrm{a} \\
(2.71)\end{array}$ & $\begin{array}{l}11.93 b \\
(3.65)\end{array}$ & $\begin{array}{l}10.41 b \\
(2.24)\end{array}$ & $\begin{array}{c}14.23 \mathrm{a} \\
(4.01)\end{array}$ & $\begin{array}{l}12.08 b \\
(4.25)\end{array}$ & $\begin{array}{l}10.33 \mathrm{c} \\
(2.53)\end{array}$ \\
\hline Angle of spatula $\left(^{\circ}\right)$ & $\begin{array}{c}52.80 \mathrm{a} \\
(2.82)\end{array}$ & $\begin{array}{c}51.99 b \\
(2.18)\end{array}$ & $\begin{array}{c}50.74 \mathrm{c} \\
(2.27)\end{array}$ & $\begin{array}{c}\mathbf{5 3 . 0 3 a} \\
(1.53)\end{array}$ & $\begin{array}{c}\mathbf{5 0 . 7 1 a} \\
(1.54)\end{array}$ & $\begin{array}{c}\mathbf{5 0 . 6 6 a} \\
(2.81)\end{array}$ & $\begin{array}{c}53.93 \mathrm{a} \\
(1.61)\end{array}$ & $\begin{array}{c}50.54 c \\
(2.19)\end{array}$ & $\begin{array}{l}51.08 \mathrm{~b} \\
(2.42)\end{array}$ \\
\hline Uniformity (-) & $\begin{array}{c}2.28 \mathrm{a} \\
(0.19)\end{array}$ & $\begin{array}{c}2.0 \mathrm{a} \\
(0.18)\end{array}$ & $\begin{array}{c}1.76 \mathrm{c} \\
(0.13)\end{array}$ & $\begin{array}{c}\mathbf{2 . 0 6 a} \\
(0.25)\end{array}$ & $\begin{array}{c}\mathbf{2 . 0 7 a} \\
(0.20)\end{array}$ & $\begin{array}{c}\mathbf{1 . 9 4 a} \\
(0.28)\end{array}$ & $\begin{array}{c}2.13 \mathrm{a} \\
(0.30)\end{array}$ & $\begin{array}{c}2.00 \mathrm{~b} \\
(0.26)\end{array}$ & $\begin{array}{l}1.91 \mathrm{c} \\
(0.21)\end{array}$ \\
\hline Angle of fall $\left(^{\circ}\right)$ & $\begin{array}{c}39.97 \mathrm{~b} \\
(1.85)\end{array}$ & $\begin{array}{c}40.88 \mathrm{a} \\
(1.71)\end{array}$ & $\begin{array}{c}37.87 \mathrm{c} \\
(4.85)\end{array}$ & $\begin{array}{c}\text { 39.39a } \\
(1.89)\end{array}$ & $\begin{array}{c}\mathbf{3 9 . 8 8 a} \\
(2.01)\end{array}$ & $\begin{array}{c}\mathbf{3 9 . 5 3 a} \\
(1.74)\end{array}$ & $\begin{array}{c}40.86 a \\
(1.61)\end{array}$ & $\begin{array}{c}39.21 b \\
(1.33)\end{array}$ & $\begin{array}{c}38.66 \mathrm{~b} \\
(4.21)\end{array}$ \\
\hline Angle of difference $\left(^{\circ}\right)$ & $\begin{array}{c}2.39 \mathrm{c} \\
(1.12)\end{array}$ & $\begin{array}{c}4.58 b \\
(2.11)\end{array}$ & $\begin{array}{c}8.09 \mathrm{a} \\
(5.17)\end{array}$ & $\begin{array}{c}\mathbf{5 . 0 7 a} \\
(3.56)\end{array}$ & $\begin{array}{c}\mathbf{5 . 4 2 a} \\
(2.51)\end{array}$ & $\begin{array}{c}\mathbf{4 . 9 2 a} \mathbf{a} \\
(1.75)\end{array}$ & $\begin{array}{r}3.59 \mathrm{c} \\
(1.67)\end{array}$ & $\begin{array}{c}4.70 \mathrm{~b} \\
(2.17)\end{array}$ & $\begin{array}{r}6.77 \mathrm{a} \\
(5.12)\end{array}$ \\
\hline Dispersibility (\%) & $\begin{array}{c}45.41 \mathrm{a} \\
(4.83)\end{array}$ & $\begin{array}{c}36.86 b \\
(5.64)\end{array}$ & $\begin{array}{c}35.85 b \\
(2.87)\end{array}$ & $\begin{array}{c}38.79 b \\
(4.98)\end{array}$ & $\begin{array}{c}46.70 \mathrm{a} \\
(2.05)\end{array}$ & $\begin{array}{c}39.95 \mathrm{a} \\
(7.37)\end{array}$ & $\begin{array}{l}37.52 \mathrm{c} \\
(5.83)\end{array}$ & $\begin{array}{c}41.06 \mathrm{a} \\
(5.81)\end{array}$ & $\begin{array}{l}37.53 \mathrm{c} \\
(6.79)\end{array}$ \\
\hline Total flood index (-) & $\begin{array}{c}67.77 \mathrm{a} \\
(4.81)\end{array}$ & $\begin{array}{c}67.10 \mathrm{~b} \\
(3.45)\end{array}$ & $\begin{array}{c}61.71 \mathrm{c} \\
(1.81)\end{array}$ & $\begin{array}{c}66.91 \mathrm{a} \\
(4.55)\end{array}$ & $\begin{array}{c}66.84 a \\
(1.54)\end{array}$ & $\begin{array}{c}64.14 b \\
(3.95)\end{array}$ & $\begin{array}{c}63.25 \mathrm{c} \\
(3.37)\end{array}$ & $\begin{array}{c}68.10 \mathrm{a} \\
(4.82)\end{array}$ & $\begin{array}{c}65.22 \mathrm{~b} \\
(4.31)\end{array}$ \\
\hline Hunter $L(-)$ & $\begin{array}{c}\mathbf{4 4 . 7 2 a} \\
(5.79)\end{array}$ & $\begin{array}{c}\mathbf{4 3 . 6 8 a} \\
(6.73)\end{array}$ & $\begin{array}{c}\mathbf{4 1 . 8 2 a} \\
(5.56)\end{array}$ & $\begin{array}{c}\mathbf{4 3 . 9 2 a} \\
(5.06)\end{array}$ & $\begin{array}{c}\mathbf{4 6 . 2 1 a} \\
(2.96)\end{array}$ & $\begin{array}{c}\mathbf{4 2 . 8 9} \mathbf{a} \\
(6.77)\end{array}$ & $\begin{array}{c}44.41 \mathrm{a} \\
(2.41)\end{array}$ & $\begin{array}{c}48.91 \mathrm{a} \\
(2.35)\end{array}$ & $\begin{array}{l}36.90 \mathrm{c} \\
(3.95)\end{array}$ \\
\hline Hunter $a(-)$ & $\begin{array}{c}9.60 \mathrm{~b} \\
(1.23)\end{array}$ & $\begin{array}{c}10.51 \mathrm{a} \\
(1.06)\end{array}$ & $\begin{array}{c}9.56 \mathrm{~b} \\
(0.65)\end{array}$ & $\begin{array}{c}\text { 10.01a } \\
(0.91)\end{array}$ & $\begin{array}{c}\mathbf{9 . 8 3 a} \\
(1.37)\end{array}$ & $\begin{array}{c}9.79 a \\
(1.81)\end{array}$ & $\begin{array}{c}9.72 b \\
(1.15)\end{array}$ & $\begin{array}{c}9.67 b \\
(0.07)\end{array}$ & $\begin{array}{c}10.31 \mathrm{a} \\
(1.26)\end{array}$ \\
\hline Hunter $b(-)$ & $\begin{array}{c}22.32 \mathrm{a} \\
(2.01)\end{array}$ & $\begin{array}{c}21.53 b \\
(1.12)\end{array}$ & $\begin{array}{c}20.16 \mathrm{c} \\
(1.21)\end{array}$ & $\begin{array}{c}\mathbf{2 1 . 8 4 a} \\
(1.81)\end{array}$ & $\begin{array}{c}\mathbf{2 1 . 9 1 a} \\
(1.56)\end{array}$ & $\begin{array}{l}\mathbf{2 0 . 8 3 a} \\
(1.5)\end{array}$ & $\begin{array}{c}20.99 a \\
(1.66)\end{array}$ & $\begin{array}{c}\mathbf{2 1 . 6 3 a} \\
(2.05)\end{array}$ & $\begin{array}{c}\mathbf{2 1 . 3 8 a} \\
(1.44)\end{array}$ \\
\hline Water activity $(-)$ & $\begin{array}{c}0.65 a \\
(0.13)\end{array}$ & $\begin{array}{c}0.43 b \\
(0.07)\end{array}$ & $\begin{array}{c}0.39 \mathrm{c} \\
(0.12)\end{array}$ & $\begin{array}{c}0.52 \mathrm{a} \\
(0.14)\end{array}$ & $\begin{array}{c}0.45 b \\
(0.03)\end{array}$ & $\begin{array}{c}0.46 b \\
(0.13)\end{array}$ & $\begin{array}{c}0.42 \mathrm{c} \\
(0.02)\end{array}$ & $\begin{array}{c}0.46 \mathrm{~b} \\
(0.01)\end{array}$ & $\begin{array}{r}0.50 \mathrm{a} \\
(0.12)\end{array}$ \\
\hline Thermal conductivity $\left(\mathrm{W} / \mathrm{m}^{\circ} \mathrm{C}\right)$ & $\begin{array}{c}0.06 \mathrm{~b} \\
(0.01)\end{array}$ & $\begin{array}{c}0.07 \mathrm{a} \\
(0.02)\end{array}$ & $\begin{array}{c}0.07 \mathrm{a} \\
(0.01)\end{array}$ & $\begin{array}{c}\mathbf{0 . 0 7 a} \\
(0.01)\end{array}$ & $\begin{array}{c}\mathbf{0 . 0 6} \mathbf{a} \\
(0.01)\end{array}$ & $\begin{array}{c}\mathbf{0 . 0 7 a} \\
(0.01)\end{array}$ & $\begin{array}{r}\mathbf{0 . 0 7 a} \\
(0.013\end{array}$ & $\begin{array}{c}\mathbf{0 . 0 7 a} \\
(0.01)\end{array}$ & $\begin{array}{c}\text { 0.08a } \\
(0.01)\end{array}$ \\
\hline Thermal resistivity $\left(\mathrm{m}^{\circ} \mathrm{C} / \mathrm{W}\right)$ & $\begin{array}{c}\mathbf{1 5 . 0 8 a} \\
(1.61)\end{array}$ & $\begin{array}{c}\mathbf{1 3 . 2 7} \mathbf{a} \\
(1.79)\end{array}$ & $\begin{array}{c}\mathbf{1 3 . 2 6 a} \\
(1.81)\end{array}$ & $\begin{array}{c}\mathbf{1 3 . 8 3 a} \\
(1.87)\end{array}$ & $\begin{array}{c}\mathbf{1 4 . 5 0 a} \\
(1.25)\end{array}$ & $\begin{array}{c}\mathbf{1 3 . 8 3 a} \\
(1.99)\end{array}$ & $\begin{array}{c}13.83 b \\
(2.41)\end{array}$ & $\begin{array}{c}14.37 \mathrm{a} \\
(1.91)\end{array}$ & $\begin{array}{c}13.42 \mathrm{~b} \\
(1.15)\end{array}$ \\
\hline Thermal diffusivity $\left(\mathrm{mm}^{2} / \mathrm{sec}\right)$ & $\begin{array}{c}0.17 \mathrm{a} \\
(0.01)\end{array}$ & $\begin{array}{c}0.15 b \\
(0.07)\end{array}$ & $\begin{array}{c}0.16 \mathrm{~b} \\
(0.11)\end{array}$ & $\begin{array}{c}0.16 \mathrm{a} \\
(0.03)\end{array}$ & $\begin{array}{l}0.16 \mathrm{ab} \\
(0.02)\end{array}$ & $\begin{array}{c}0.15 \mathrm{a} \\
(0.02)\end{array}$ & $\begin{array}{c}0.17 \mathrm{a} \\
(0.01)\end{array}$ & $\begin{array}{c}0.15 b \\
(0.01)\end{array}$ & $\begin{array}{c}0.15 b \\
(0.03)\end{array}$ \\
\hline Porosity (-) & $\begin{array}{r}8.94 a \\
(1.93)\end{array}$ & $\begin{array}{c}9.01 \mathrm{a} \\
(1.95)\end{array}$ & $\begin{array}{c}7.96 a \\
(2.37)\end{array}$ & $\begin{array}{c}\mathbf{8 . 7 2 a} \\
(1.96)\end{array}$ & $\begin{array}{c}\text { 8.55a } \\
(1.85)\end{array}$ & $\begin{array}{c}\mathbf{8 . 5 5 a} \\
(2.29)\end{array}$ & $\begin{array}{c}\mathbf{8 . 2 6 a} \\
(1.85)\end{array}$ & $\begin{array}{c}\mathbf{9 . 1 6 a} \\
(2.42)\end{array}$ & $\begin{array}{c}\text { 8.51a } \\
(2.08)\end{array}$ \\
\hline
\end{tabular}

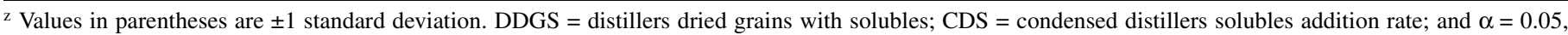
LSD. Bold font indicates that there is no significant difference among levels for a given independent variable for that property. 
fined as the ratio of PBD to ABD. Jenike (1964) flow indices for this particular study were calculated from the previously derived regression equation involving similar CDS and drying temperature levels. More details on this regression equation and its derivation can be found in Bhadra et al (2009c).

\section{Statistical Analyses}

Formal statistical data analyses were completed with Microsoft Excel 2003 (Redmond, WA, U.S.A.) and SAS software (version 8, SAS Institute, Cary, NC, U.S.A.). Analyses included summary statistics and least significant difference (LSD) testing at the $95 \%$ confidence level (i.e., $\alpha=0.05$ ) to determine differences and interaction effects between the treatment combinations for each drying temperature, cooling temperature, and CDS level. Correlation analysis (PROC CORR) among all properties was performed to examine linear relationships between the properties at the $95 \%$ significance (i.e., $\alpha=0.05$ ) level. TableCurve 3D software (version 4.0.01, SYSTAT Software, San Jose, CA, U.S.A.) was then used to develop regression equations for response surfaces to analyze selected dimensionless flow parameters as a function of varying CDS levels $(\%, w b)$ and cooling temperature/drying temperature ratios (-). Partial least squares (PLS) modeling, principal component analysis (PCA), cluster analysis (CA), and relevant multivariate analyses were performed with Minitab software (version 14, Minitab, State College, PA, U.S.A.).

\section{RESULTS AND DISCUSSION}

\section{Main Effects and Interactions Effects}

Table I provides a summary of main effect analysis for all the Carr (1965) and Jenike (1964) flow properties. When the LSD test was implemented, significant differences in the main effects for drying temperature levels $\left(100,200\right.$, and $\left.300^{\circ} \mathrm{C}\right)$, cooling temperature levels $\left(-12,25\right.$, and $\left.35^{\circ} \mathrm{C}\right)$, and CDS addition levels $(10,15$, and $20 \%$, wb) were observed. The flowability of DDGS is a multivariate phenomenon and is strongly affected by the variability in the properties, even though DWG and CDS were collected from the same ethanol plant. Differences during mixing of the wet cake with CDS and the drying operation can bring more significant changes in physical and flowability properties in DDGS than the variations in corn quality (Kleinschmit et al 2006). These results verified our hypothesis that drying temperatures, cooling temperatures, and CDS addition levels created significant differences in DDGS physical and flow properties. Table I shows that for 12 flow properties (indicated in bold fonts) no significant differences among the cooling temperature levels were reported. However, for the main effect of CDS level, only four flow properties (indicated in bold fonts) showed no significant differences among their levels, and for drying temperature it was three flow properties. Thus, the overall flowability problem of DDGS comes mainly from the variability caused by drying temperature and CDS levels, and it is less affected by cooling temperature. Perhaps our selected cooling temperature levels were not wide enough to account for the significant differences in the flow properties. A study done by Bhadra et al (2009c) showed similar results of significant differences for drying temperatures and CDS levels. However, there were no reports on the main effect of cooling temperatures. The cooling temperature levels $\left(-12\right.$ and $\left.35^{\circ} \mathrm{C}\right)$ were decided based upon the average cold and hot temperature reports in the Midwest United States.

Table II indicates significant interaction effects between drying temperatures, cooling temperatures, and CDS levels for more than $50 \%$ of the flowability properties. Thus, drying temperature, cooling temperature, and CDS variation can synergistically affect the flowability of DDGS. For interaction effects between only drying temperature and CDS level, we could see that Hunter $a$, water activity, porosity, and PDI did not show any significance. However, our previous study on DDGS flowability with varying drying temperature and CDS levels showed nonsignificant interaction effects only for HR and $C_{\mathrm{c}}$ (Bhadra et al 2009c).

\section{Property Relationships}

Table III illustrates Pearson product moment linear correlation analyses (Speigel 1994) performed for all the flow and physical properties. The correlation coefficient for a particular combination determines how closely the two properties are related to each other in a linear relationship. Results showed only seven combinations had $P$ values less than $\alpha=0.05$ (i.e., were significant correlations) and also had $R^{2}$ values of $\geq 0.36$. Out of these seven significant combinations, four variable combinations had $R^{2}$ values from 0.36 to 0.49 , two variable combinations had $R^{2}$ values from 0.5 to 0.8 , and one combination had an $R^{2}$ value from 0.9 to 1.0 .

TABLE II

Interaction Effects ( $P$ Values) Due to Drying Temperature, Cooling Temperature, and CDS Level on Flow and Physical Properties of DDGS ${ }^{z}$

\begin{tabular}{|c|c|c|c|c|c|c|c|}
\hline Property & CT & DT & $\mathbf{C T} \times \mathbf{D T}$ & CDS & $\mathrm{CT} \times \mathrm{CDS}$ & $\mathrm{CDS} \times \mathrm{DT}$ & $\mathrm{CT} \times \mathrm{DT} \times \mathrm{CDS}$ \\
\hline Angle of repose $\left(^{\circ}\right)$ & 0.0021 & 0.0019 & $<0.0001$ & 0.0225 & 0.0991 & $<0.0001$ & $<0.0001$ \\
\hline Aerated bulk density $\left(\mathrm{kg} / \mathrm{m}^{3}\right)$ & 0.8300 & $<0.0001$ & 0.1330 & 0.0003 & 0.8777 & 0.0330 & 0.0566 \\
\hline Particle size $\left(d_{\mathrm{gw}}, \mathrm{mm}\right)$ & 0.0030 & $<0.0001$ & 0.1110 & $<0.0001$ & 0.0388 & $<0.0001$ & 0.0045 \\
\hline Total flow index (-) & 0.0050 & 0.0002 & 0.0400 & 0.0190 & 0.0018 & $<0.0001$ & $<0.0001$ \\
\hline Jenike flow function index $(-)$ & $<0.0001$ & 0.7900 & 0.1600 & 0.7600 & 0.0066 & 0.0006 & 0.0483 \\
\hline Protein dispersibility index (\%) & 0.0040 & $<0.0001$ & 0.0014 & $<0.0001$ & 0.7770 & 0.0868 & $<0.0001$ \\
\hline Compressibility $(\%)$ & $<0.0001$ & $<0.0001$ & $<0.0001$ & $<0.0001$ & $<0.0001$ & $<0.0001$ & 0.0072 \\
\hline Angle of fall $\left(^{\circ}\right)$ & 0.8600 & $<0.0001$ & 0.2450 & $<0.0001$ & 0.0833 & $<0.0001$ & 0.0051 \\
\hline Angle of difference $\left(^{\circ}\right)$ & 0.7692 & $<0.0001$ & 0.0200 & $<0.0001$ & 0.2700 & $<0.0001$ & 0.1600 \\
\hline Dispersibility (\%) & 0.0212 & $<0.0001$ & 0.0002 & $<0.0001$ & $<0.0001$ & $<0.0001$ & $<0.0001$ \\
\hline Total flood index(-) & $<0.0001$ & $<0.0001$ & $<0.0001$ & $<0.0001$ & $<0.0001$ & $<0.0001$ & $<0.0001$ \\
\hline Hunter $L(-)$ & 0.1110 & 0.0022 & 0.6436 & $<0.0001$ & 0.0160 & 0.0199 & 0.1300 \\
\hline Hunter $a(-)$ & 0.3300 & 0.0018 & 0.8270 & 0.0460 & 0.3100 & 0.0920 & 0.4620 \\
\hline Hunter $b(-)$ & 0.0023 & $<0.0001$ & 0.2930 & 0.2600 & 0.9437 & 0.0100 & 0.4050 \\
\hline
\end{tabular}

${ }^{\mathrm{z}}$ DDGS $=$ distillers dried grains with solubles; $\mathrm{CT}=$ cooling temperature $\left({ }^{\circ} \mathrm{C}\right)$; DT $=$ drying temperature $\left({ }^{\circ} \mathrm{C}\right)$; CDS $=$ condensed distillers solubles addition levels (\%,wb); and $\alpha=0.05$. 
Thermal conductivity had high correlation with thermal resistivity. AoR, an important parameter for flowability, showed reasonable correlation with uniformity. AoR was strongly dependent on the particle size and shape. Uniformity was also a parameter that depended on the size of the particles. Thus, correlation between AoR and uniformity was logical. Jenike flow index had a correlation with porosity. This seemed logical, as both porosity and Jenike flow index measured the bulk handling ability of the material. Particle porosity was closely linked with particle size and contributed to the overall flowability of the material. Again, particle size had a strong relationship to bulk density, and changes in density could affect the stress developed in bulk or particulate materials. Because of stress, compression of the ma-

TABLE III

Significant $(P<0.05)$ Pearson Linear Correlation Coefficients $(r)$ Between Flow and Physical Properties for DDGS Prepared Using Varying Drying Temperature, Cooling Temperature, and CDS Levels ${ }^{\mathrm{Z}}$

\begin{tabular}{lccc}
\hline Property Relationship & $\boldsymbol{r}$ Value & $\boldsymbol{R}^{\mathbf{2}}$ & $\boldsymbol{P}$ Value \\
\hline Thermal conductivity $\times$ thermal resistivity & -0.9917 & 0.98 & $<0.0001$ \\
AoF $\times$ AoD & -0.8444 & 0.71 & $<0.0001$ \\
Porosity $\times$ Jenike flow index & -0.7033 & 0.49 & $<0.0001$ \\
AoR $\times$ PDI & -0.6767 & 0.45 & $<0.0001$ \\
AoR $\times$ uniformity & -0.6143 & 0.37 & $<0.0001$ \\
Thermal conductivity $\times$ dispersibility & -0.6072 & 0.36 & $<0.0001$ \\
PDI $\times$ uniformity & 0.8294 & 0.69 & $<0.0001$ \\
\hline
\end{tabular}

${ }^{\mathrm{z}}$ DDGS $=$ distillers dried grains with solubles; AoR = angle of repose; $\mathrm{AoF}=$ angle of fall; $\mathrm{AoD}=$ angle of difference; PDI = protein dispersibility index; and $\mathrm{CDS}=$ condensed distillers solubles addition rate $(\%, \mathrm{wb})$. terial occurs, affecting yield strength and stress. Therefore, Jenike flow index and porosity were interrelated and affected the overall flowability.

\section{Effects of Drying Temperature, Cooling Temperature, and CDS Levels on AoR}

Figure 2 shows the difference in the AoR $\left({ }^{\circ}\right)$ values resulting from drying temperature rather than cooling temperature. Figure 2 did not predict major differences in AoR for cooling temperatures of $-12,25$, and $35^{\circ} \mathrm{C}$. For all CDS addition levels, the AoR varied from 35 to $47^{\circ}$, and according to Carr classification, DDGS samples belonged to the "passable to fair" category. AoR $>48^{\circ}$ indicated poor flow in biomaterials, whereas $<30^{\circ}$ indicated better flow (Carr 1965; Bhadra et al 2009a). AoR is believed to be dependent on particle size and shape. Coarser particles typically yield higher AoR. For DDGS, however, AoR did not show such exponential decrease; rather, we could observe a linear change with drying temperature (Fig. 2).

\section{Effects of Drying Temperature, Cooling Temperature, and CDS Levels on ABD $\left(\mathrm{kg} / \mathrm{m}^{3}\right)$}

Figure 2 shows the effects of drying and cooling temperatures on ABD of the DDGS samples at different CDS addition levels. With an increase in the drying temperature from 100 to $300^{\circ} \mathrm{C}$, ABD slightly increased for 15 and $20 \%$ (wb) CDS levels. Unlike our previous research, this study did not produce significant interaction effects between CDS level, drying temperature, and cooling temperature for ABD (Bhadra et al 2009c). According to Chegini and Ghobadian (2007), particle size increased with
$10 \%(w b) \operatorname{CDS}$

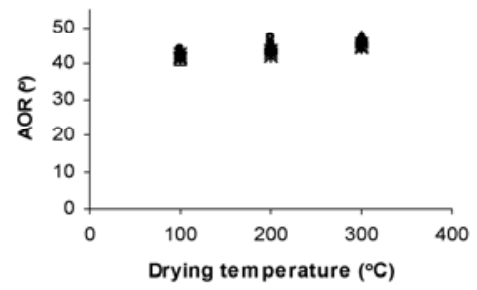

$10 \%(w b) \operatorname{CDS}$

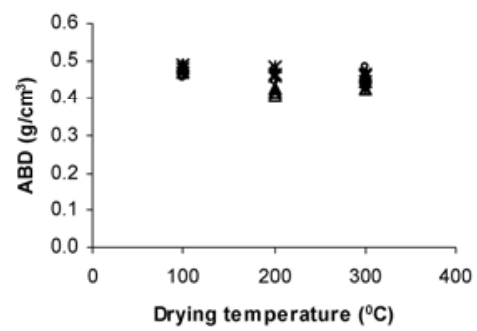

$10 \%(w b) \operatorname{CDS}$

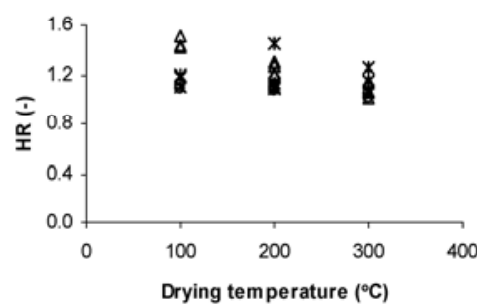

$15 \%$ (wb) CDS

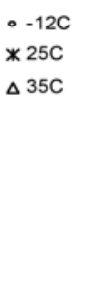

$-12 \mathrm{C}$

$* 25 \mathrm{C}$

$\Delta 35 \mathrm{C}$

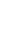

$15 \%(w b) \operatorname{CDS}$
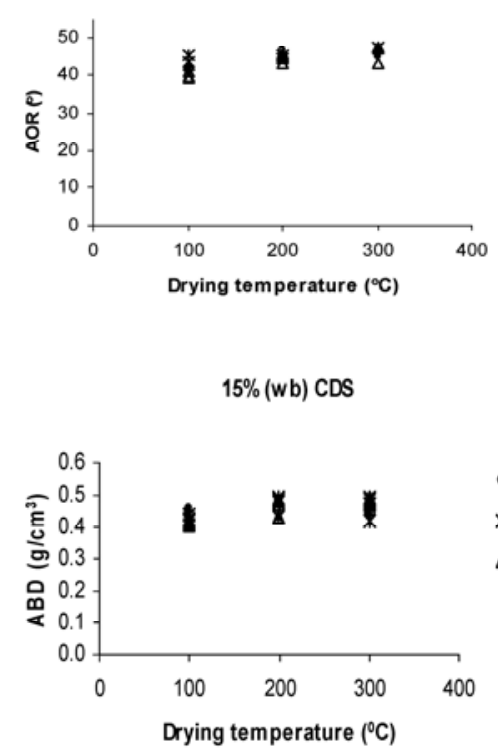

$15 \%(w b) \operatorname{CDS}$
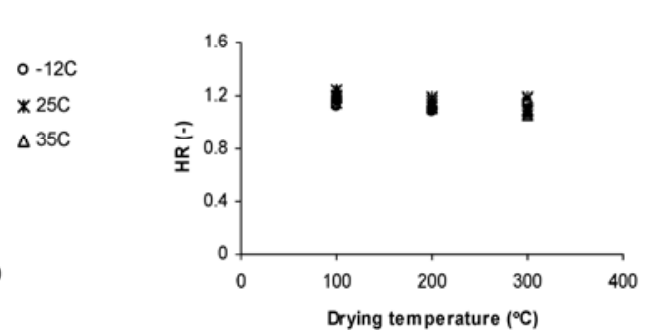
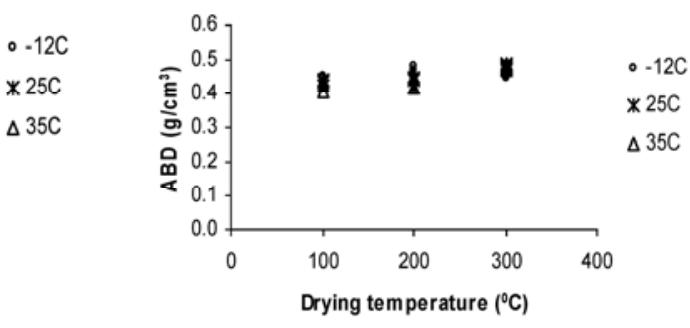

$20 \%(w b)$ CDS

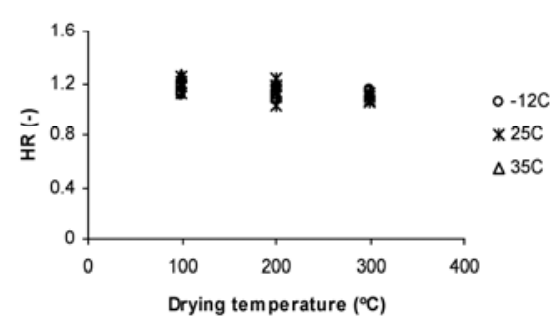

Fig. 2. Relationships between selected properties, drying temperature $\left({ }^{\circ} \mathrm{C}\right)$, and cooling temperature $\left({ }^{\circ} \mathrm{C}\right)$, according to condensed distillers solubles (CDS) level (\%, wb). Selected properties are angle of repose (AOR), aerated bulk density (ABD), and Hausner ratio (HR). 
ABD. This increment resulted from the rapid formation of a dried layer on the particle surfaces. Hence, particle size increase resulted from case hardening of the droplets at the higher temperatures. This phenomenon led to the formation of vapor-impermeable films on the particle surface, followed by the formation of vapor bubbles and, consequently, droplet expansion. Hardened skin did not allow moisture to exit from the surface, and as a consequence the particle size increased. Thus, increase in particle size affected the ABD and compressibility of food particulates, and hence, flow problems were observed. Like AoR, ABD did not yield any major difference between the three cooling temperatures. Furthermore, time consolidation of particulates and its effects on bulk density were measured by Teunou and Fitzpatrick (2000), but our current study did not examine these effects.

\section{Effects of Drying Temperature, Cooling Temperature, and CDS Levels on HR (-)}

Figure 2 presents HR (-) values (ranging from 1.03 to 1.44 ) for DDGS with varying drying temperature, cooling temperature, and CDS levels. Knowledge of the HR is utilized for understanding the sieving mechanism and compaction of particulates at the initial stage (Grey and Beddow 1969). Higher HR (>1.25) indicated poor flowability in the DDGS samples (Aulton 2001). For DDGS, HRs were mostly below 1.25 . However, for $10 \%$ CDS levels, the HRs were higher than 1.25 in some cases (Fig. 2). A higher CDS level implies a higher fat level in DDGS because CDS contains higher proportions of lipids and fat components. Fitzpatrick et al (2004) showed that increase in fat levels could create potential flow problems in milk particles. However, for DDGS we did not see such results, and we got slightly lower HR values for CDS

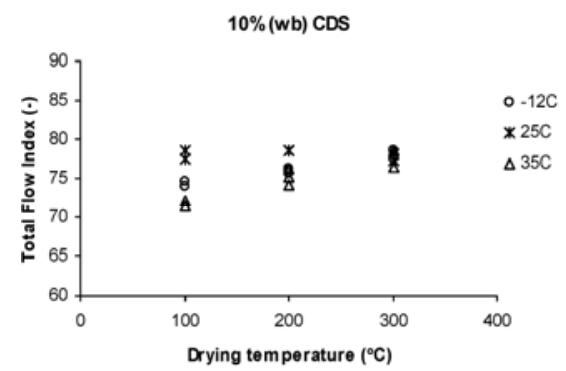

$10 \%$ (wb) CDS

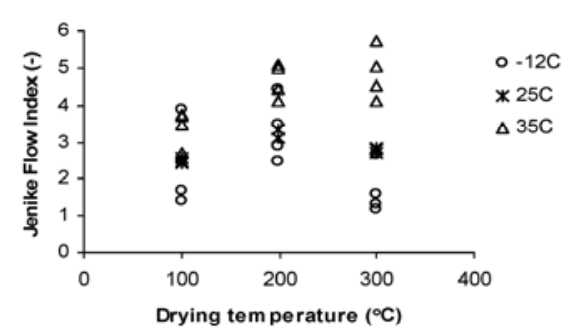

$10 \%$ (wb) CDS

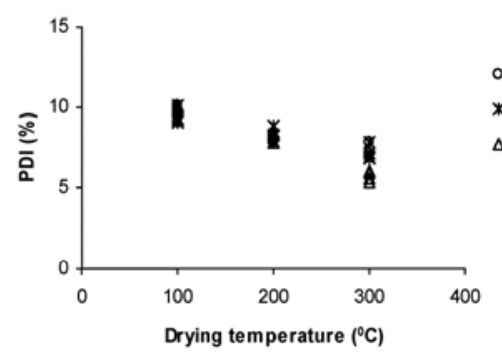

$10 \%$ (wb) CDS

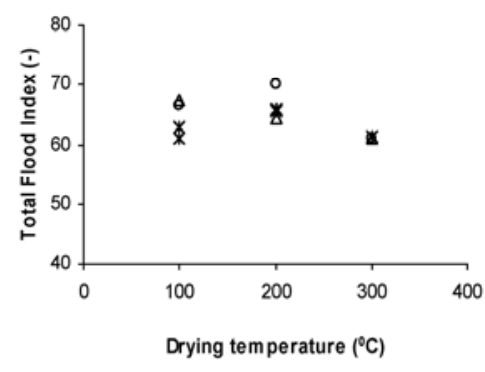

$15 \%$ (wb) CDS

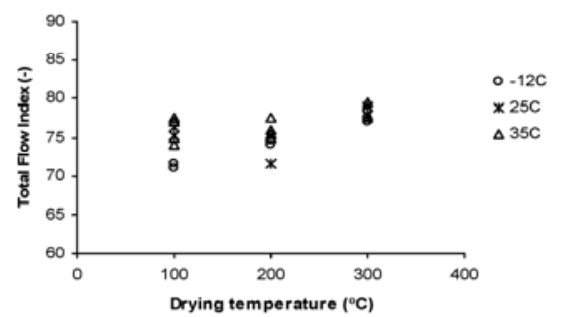

$15 \%(w b) \operatorname{CDS}$

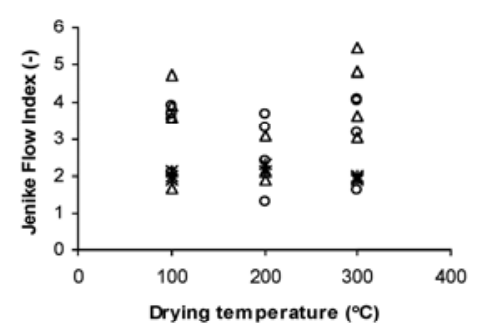

$15 \%$ (wb) CDS

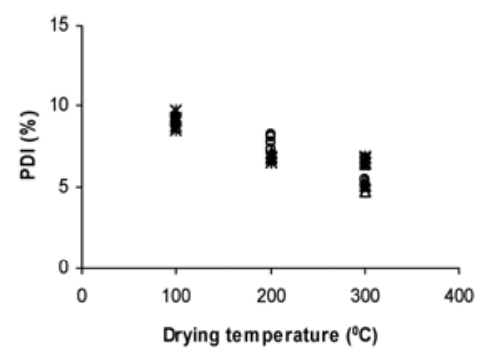

$15 \%$ (wb) CDS

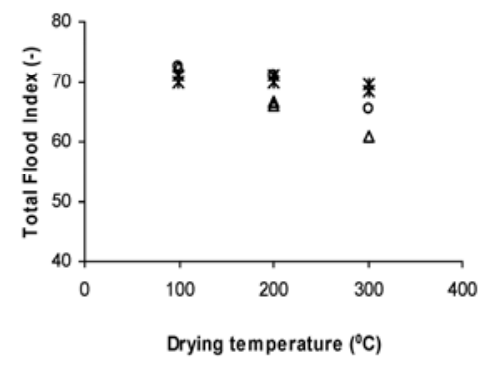

$20 \%$ (wb) $\operatorname{CDS}$

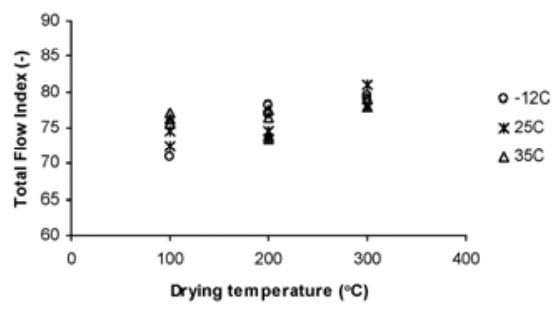

$20 \%$ (w b) CDS

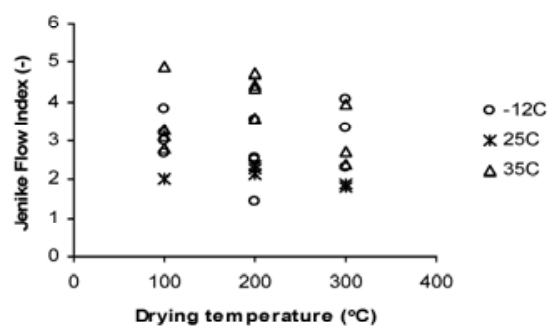

$20 \%$ (wb) CDS

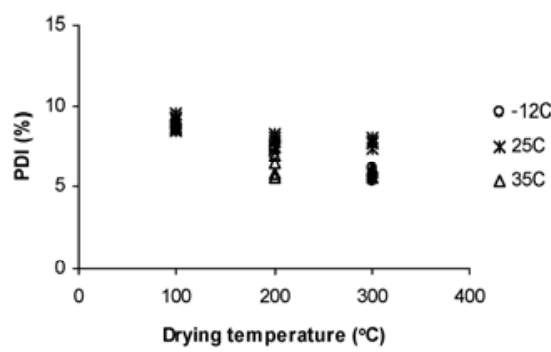

$20 \%$ (wb) CDS

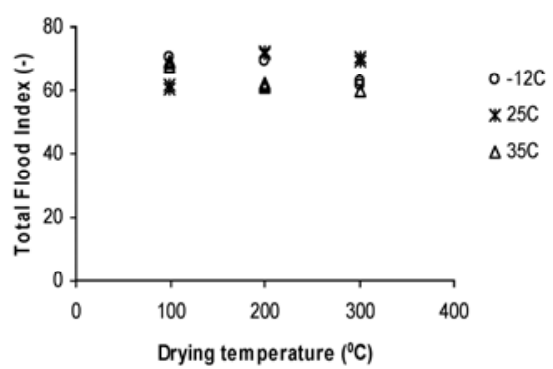

Fig. 3. Relationships between selected properties, drying temperature $\left({ }^{\circ} \mathrm{C}\right)$, and cooling temperature $\left({ }^{\circ} \mathrm{C}\right)$, according to condensed distillers solubles (CDS) level $(\%, w b)$. Selected properties are total flow index, Jenike flow index, protein dispersibility index (PDI), and total flood index. 
levels of 15 and $20 \%$ (wb). Higher fat levels can possibly help in lubrication of the DDGS samples, and thus, the HR values decreased, indicating slightly better flowability characteristics. Similar results were seen in the study done by Ganesan et al (2007a, 2007b) with DDGS samples. Also, HR did not show prominent differences between the three cooling temperatures. However, there were clear distinctions in the HRs for varying drying temperatures. It was observed that increase in the drying temperature decreased the HR. This decrease in HR with the increase in drying temperature was similar to the results observed in the previous study by Bhadra et al (2009c).

\section{Effects of Drying Temperature, Cooling Temperature, and CDS Levels on Total Flowability Index (-)}

Figure 3 presents total flowability index (-) behavior with varying CDS levels, drying temperatures, and cooling temperatures. The total flowability index (-) was found to range from 71.00 to 80.25. This range was categorized as "fair to good" by Carr (1965). Carr (1965) total flowability index (-) was the sum of AoR, ABD, PBD, compressibility, AoS, and uniformity, providing a comprehensive measurement of flowability in food particulate substances (Bhadra et al 2009a). As shown in Figure 3, there were no major differences among the cooling temperatures $(-12,25$, and $35^{\circ} \mathrm{C}$ ); however, there were prominent differences among the drying temperatures for all the CDS levels. Higher total flowability index indicated better flow in particulates. As we increased the drying temperatures, total flowability indices slightly increased for all the CDS levels. Thus, increased drying temperatures would improve the flowability of DDGS but would also increase the overall drying cost in the industry. Therefore, ethanol plants need to optimize drying temperatures to achieve better flowability for a given cooling condition and CDS addition level.

\section{Effects of Drying Temperature, Cooling Temperature, and CDS Levels on Jenike Flow Index (-)}

Figure 3 presents the flowability behavior of prepared DDGS samples for various CDS and drying temperature levels based on the Jenike (1964) flow index. The Jenike flow indices for -12 and $35^{\circ} \mathrm{C}$ were obtained from the multivariate PLS regression equa- tion derived from our previous study with similar CDS and drying temperature levels (Bhadra et al 2009c). But the Jenike flow indices for $25^{\circ} \mathrm{C}$ were the actual observed data obtained from a stressstrength Mohr circle diagram, from our previous study with similar drying temperature and CDS levels (Bhadra et al 2009c). Thus, Figure 3 represents the overall comprehensive data for Jenike flow index $(\approx 1.0-6$. 0$)$ for all three cooling temperatures $\left(-12,25\right.$, and $\left.35^{\circ} \mathrm{C}\right)$. Scatter in the dataset was observed, possibly because the indices were calculated from regression equations for -12 and $35^{\circ} \mathrm{C}$ and were observed values using the Jenike (1964) shear test procedure for $25^{\circ} \mathrm{C}$. No definitive patterns in the Jenike flow index were observed for all CDS levels. However, our previous study with only CDS and drying temperature indicated a definite trend in flow function curves (Bhadra et al 2009c). Similar research was done by Fitzpatrick et al (2004) on different types of milk particle samples varying in storage temperatures and fat content. The Jenike flow index ranged from 1.74 to 6.3 , depending on fat content of the milk particles. The Jenike flow index decreased about $30 \%$ as the storage temperature increased from 5 to $25^{\circ} \mathrm{C}$, indicating good flow. However, in the current study, no such definitive trends were found for DDGS samples. Most DDGS samples yielded a Jenike flow index of $<4$. According to Thomas and Schubert (1979), a Jenike flow index of $<4$ indicates cohesive characteristics in the particles. Thus, for this study, most of the DDGS samples showed potential flow problems, because Jenike flow indices were mostly <4 (Fig. 3).

\section{Effects of Drying Temperature, Cooling Temperature, and CDS Levels on PDI (\%)}

From Figure 3, we can clearly observe a decrease in the PDI with an increase in the drying temperature for the DDGS samples prepared at various CDS levels. PDI gives an estimate of the amount of water-soluble protein present in the sample. Similar results of decreasing PDI with increasing drying temperature were obtained by Thomas et al (1997) and Qin et al (1998). In Thomas et al (1997), the decrease in the PDI level was linear with an increase in the drying temperature for soy grits, similar to what was found in this study. However, for Qin et al (1998) the decrease in PDI was exponential for full-fat soybeans col-

TABLE IV

Prediction Models for Selected Dependent Variables Developed by Response Surface Regression ${ }^{\mathrm{z}}$

\begin{tabular}{|c|c|c|c|c|}
\hline \multirow[b]{2}{*}{ Model Components } & \multicolumn{4}{|c|}{ Dependent Variable } \\
\hline & $\begin{array}{l}\text { Angle of Repose } \\
\left({ }^{\circ}\right)\end{array}$ & $\begin{array}{c}\text { Hausner Ratio } \\
(-)\end{array}$ & $\begin{array}{c}\text { Hunter } L \\
(-)\end{array}$ & $\begin{array}{l}\text { Total Flowability Index/ } \\
\text { Total Floodability Index (-) }\end{array}$ \\
\hline$x$ & $\mathrm{CT} / \mathrm{DT}$ & $\mathrm{CT} / \mathrm{DT}$ & $\mathrm{CT} / \mathrm{DT}$ & $\mathrm{CT} / \mathrm{DT}$ \\
\hline$y$ & CDS & CDS & CDS & CDS \\
\hline Prediction equation & $z=a+b x+c x^{2}+d y+e y^{2}$ & $z=a+b x^{2}+c e^{-y}$ & $z=a+b e^{y / w y}+(c y / \ln y)$ & $\begin{aligned} z= & a+b x+(c / y)+d x^{2}+\left(e / y^{2}\right)+(f x / y)+g x^{3} \\
& +\left(h / y^{3}\right)+\left(i x / y^{2}\right)+\left(j x^{2} / y\right)\end{aligned}$ \\
\hline Adjusted $R^{2}$ & 0.53 & 0.57 & 0.68 & 0.59 \\
\hline$F$ statistic & 21.26 & 50.05 & 80.21 & 12.90 \\
\hline Standard error & 1.47 & 0.06 & 3.31 & 0.05 \\
\hline \multicolumn{5}{|l|}{ Model parameters } \\
\hline$a$ & 52.84 & 1.08 & $20,160.67$ & 2.02 \\
\hline$b$ & 11.93 & 1.70 & $-18,635.76$ & 1.71 \\
\hline$c$ & -64.69 & 569.93 & -878.44 & -25.60 \\
\hline$i$ & & & & -70.09 \\
\hline$j$ & & & & 92.68 \\
\hline wy & & & -74.71 & \\
\hline Figure number & 4 & 5 & 6 & 7 \\
\hline
\end{tabular}

${ }^{\mathrm{z}} z=$ dependent variable; $\mathrm{CDS}=$ condensed distillers solubles addition rate $(\% \mathrm{wb}) ; \mathrm{CT}=$ cooling temperature $\left(^{\circ}\right)$; and $\mathrm{DT}=$ drying temperature $\left({ }^{\circ}\right)$. 
lected from different origins. The decrease in PDI resulted from denaturation of the protein at high temperatures, and hence, changes in the protein's solubility properties. Chell (1992) observed that heat-processed soy flour had lower PDI values but high nutritional content and, thus, was a better product for consumption and marketability. Additionally, we could observe no prominent differences in the PDI values among the three cooling temperature levels.

In terms of flowability, protein side chains may facilitate the formation of hydrophilic bonds with the associated moisture film present in between the particles, therefore decreasing the overall flowability because of mutual attraction between the particles. But increase in the drying temperature causes denaturation of protein, because of which the hydrophillicity changes, resulting in no attraction between particles. Soybean meals and untreated soybean samples collected from different zones in the United States showed PDI values from 24.3 to $31 \%$. PDI values of raw soybeans were found to be around $85 \%$ by a group of researchers (Chell 1992; Qin et al 1998). This range of PDI (for soybeans) was much higher than our observed values of PDI for DDGS (5.92-9.12\%). This occurred because soybeans have higher protein content than DDGS. Thomas et al (1997) reported that PDI was a better parameter than nitrogen solubility index to discriminate between treatments and process parameters. Moreover, non-

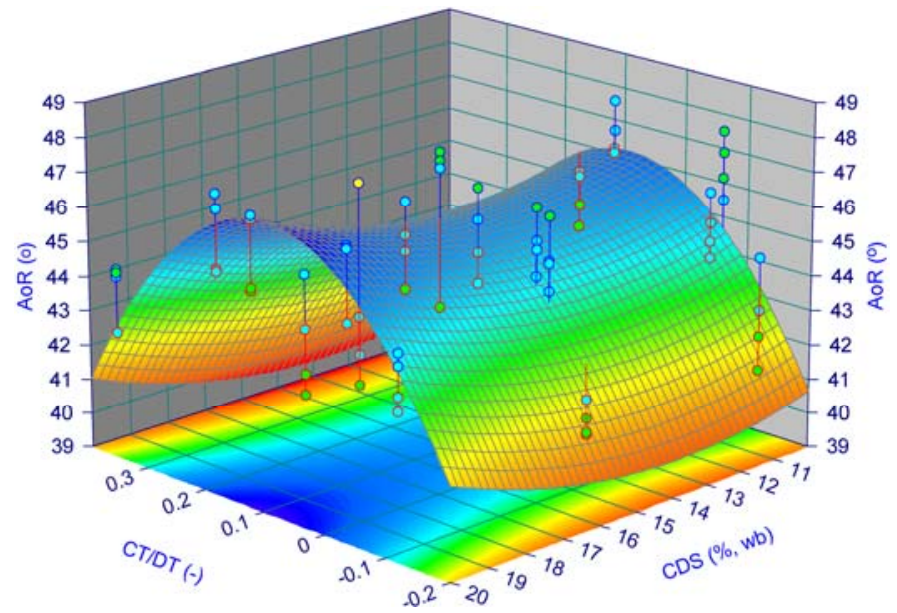

Fig. 4. Response surface plot for angle of repose $\left(\mathrm{AoR},{ }^{\circ}\right)$ as a function of cooling temperature (CT)/drying temperature (DT) ratio (-) and condensed distillers solubles (CDS) level (\%,wb).

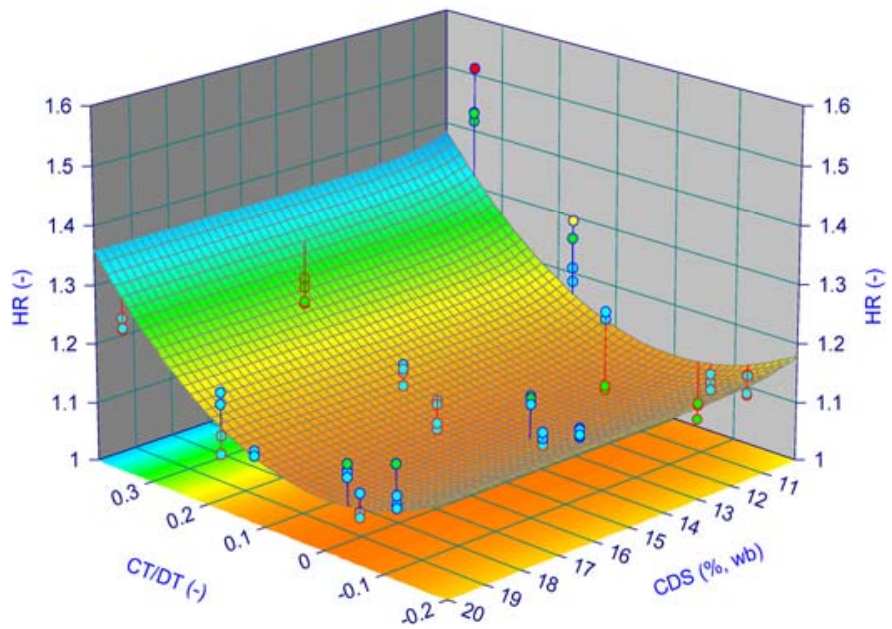

Fig. 5. Response surface plot for Hausner ratio (HR, -) as a function of cooling temperature (CT)/drying temperature (DT) ratio (-) and condensed distillers solubles (CDS) level $(\%, w b)$. protein nitrogen was also not desired in our experimental goals, so we selected PDI to study.

\section{Response Surface Regression}

Table IV indicates the nonlinear regression output of the various selected flow parameters, as shown in Figures 4-7, in order to design a predictive model for understanding DDGS flowability due to dependence on cooling temperature, drying temperature, and CDS levels. The dependent variables considered were the Carr and Jenike test properties, PDI, thermal properties, particle size, and porosity (a list of all the properties is given in Table I), and the independent variables were CDS addition levels (10, 15, and $20 \%$, wb), cooling temperatures $\left(-12,25\right.$, and $\left.35^{\circ} \mathrm{C}\right)$, and drying temperatures $\left(100,200\right.$, and $\left.300^{\circ} \mathrm{C}\right)$. Based on statistical output (Table IV), we clearly saw that the Hunter $L(-)$ scale gave the highest $R^{2}$ of 0.70 with a standard error value of 3.31. However, the ratio of total flow index/total flood index (-) yielded a slightly lower $R^{2}$ value $(0.65)$ but had the lowest standard error value (0.05) compared with other reported dimensionless parameters in Table IV. Thus, from a standard error point of view, the parameter total flowability index/total floodability index $=f$ (cooling temperature/drying temperature and CDS levels) resulted in a better model for flowability than Hunter $L(-)=f$ (cooling temperature/drying temperature and CDS levels).

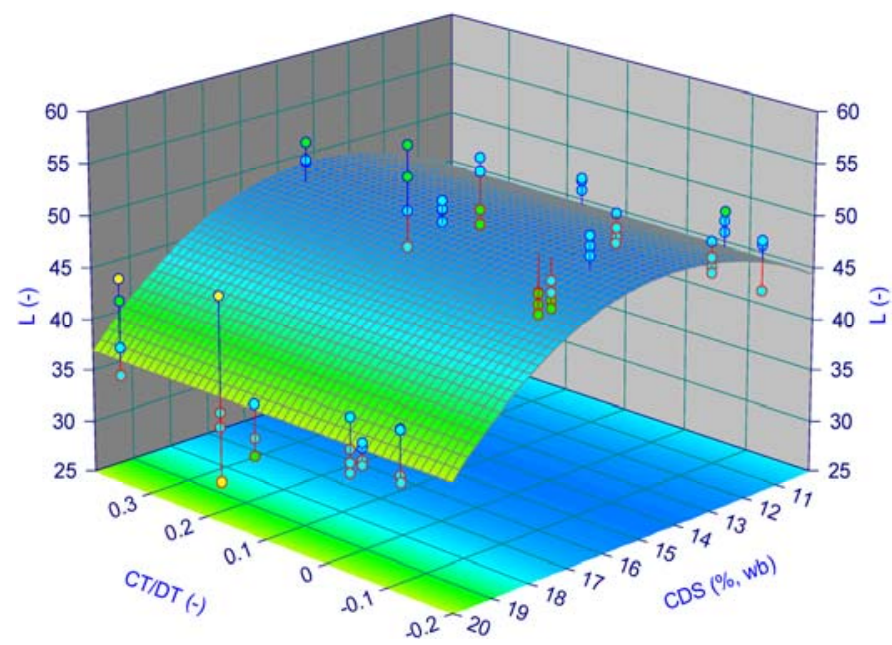

Fig. 6. Response surface plot for Hunter $L(-)$ as a function of cooling temperature (CT)/drying temperature (DT) ratio (-) and condensed distillers solubles (CDS) level (\%,wb).

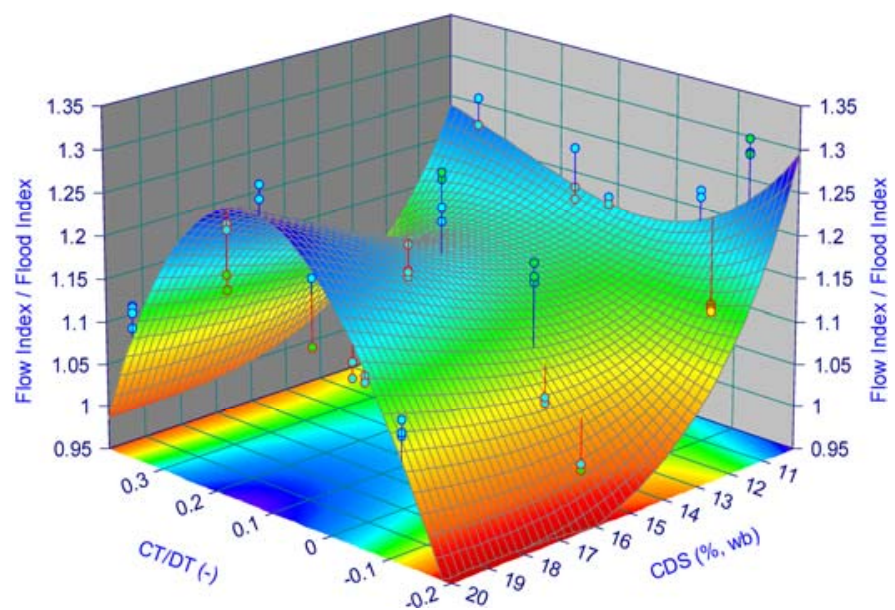

Fig. 7. Response surface plot for total flow index/total flood index ratio $(-)$ as a function of cooling temperature (CT)/drying temperature (DT) ratio (-) and condensed distillers solubles (CDS) level (\%, wb). 
Moreover, considering our objective to develop a comprehensive flowability model for dimensionless flow parameters with varying cooling temperature, drying temperature, and CDS levels, the Hunter $L(-)$ color scale index was not a very adequate indicator. The Hunter $L(-)$ color scale represents darkness of the color in the DDGS samples (Hunter Associates Laboratory 2002). Higher values of $L(-)$ represent lighter-colored samples. As shown in Figure 6 (response surface plot), the highest $L(-)$ values were found for $15 \%(\mathrm{wb})$ CDS levels. Additionally, from Figure 6 and Table IV, it was very clear that Hunter $L(-)$ did not show any dependence on cooling and drying temperature levels. Also, the parameter estimates generated from the modeling yielded large and complicated numbers, which would create problems with future computations. However, Cromwell et al (1993) indicated that color values were correlated with the nutritional components of the biomaterial. Dark-colored DDGS showed lower nutritional values than light-colored DDGS samples, because the overheating of products caused binding of lysine in the Maillard reaction and partial destruction of cystine. But we expected drying and cooling temperatures to significantly impact color values, which was not the case.

From Table IV (Fig. 7), we observed that the total flow index/total flood index $(-)\left(R^{2}=0.65\right.$, standard error $\left.=0.05\right)$ yielded a long and complicated regression equation, which was not at all practical for future flowability predictions for industrial uses. Other key flowability parameters like AoR and HR did not provide regression equations with $R^{2}$ values higher than 0.60 . Thus, it was not possible to conclude that HR and AoR would be adequate prediction parameters for flowability analysis of DDGS with varying drying temperature, cooling temperature, and CDS levels. Previous study with similar CDS levels and drying temperatures yielded much higher $R^{2}$ values for total flow index/total flood index (0.92), total flow index/Jenike flow index (0.94), AoR
(0.88), and Jenike flow index (0.84) (Bhadra et al 2009c). However, for this study we could not achieve such high $R^{2}$ ranges for the abovementioned dimensionless flow parameters. Perhaps cooling and drying temperatures interacted simultaneously along with CDS levels to provide a different response in this study.

Because our overall objective of finding a comprehensive model of DDGS flowability with varying cooling temperature, drying temperature, and CDS levels was not successful using response surface regression models, we proceeded toward multivariate regression modeling based on PCA and PLS.

\section{Multivariate Analysis}

Multivariate regression analysis can be an extension of the multiple linear regression models or the general stepwise regression model. This procedure is effective in providing a relationship between response variables (dependent variables) and predictors (independent variables). PLS regression is a more advanced multivariate regression program based on principal components. The principal components are a set of new uncorrelated variables that adequately represent the complete original set of correlated dependent variables (Johnson 1998). The formation of the principal components is based on either a sample variance or a correlation matrix. The new variables are formed by linear combinations of the original mean-corrected variables and eigenvectors produced from PCA (Sharma 1996). According to Johnson (1998), multivariate analysis is extremely effective when multicollinearity exists among the variables. For this study, correlation among the dependent variables and the interaction effects among the independent variables were significant. Carr flow properties, physical properties, and Jenike properties were sometimes highly correlated between each other. Correlation between Carr flow properties, physical properties, and Jenike properties for DDGS samples was reported by Bhadra et al (2009c, 2009d). Moreover, PCA helps to screen

TABLE V

Parameter Estimates from Partial Least Squares (PLS) Regression for Selected Response Variables, as a Multivariate Function of All Other Flow and Physical Properties

\begin{tabular}{|c|c|c|c|}
\hline \multirow[b]{2}{*}{ Predictor Variables } & \multicolumn{3}{|c|}{ Response Variable } \\
\hline & Angle of Repose $\left(^{\circ}\right)$ & Jenike Flow Index (-) & Total Flow Index/Jenike Flow Index (-) \\
\hline Constant & 5.12 & 9.64 & -51.86 \\
\hline Condensed distillers solubles addition rate $(\%, \mathrm{wb})$ & -0.02 & 0.04 & 0.11 \\
\hline Cooling temperature/drying temperature $(-)$ & -0.56 & 2.70 & -37.75 \\
\hline Hunter $L(-)$ & -0.02 & 0.02 & -0.20 \\
\hline Hunter $a(-)$ & -0.01 & 0.01 & -0.26 \\
\hline Hunter $b(-)$ & 0.07 & 0.01 & -0.53 \\
\hline Thermal conductivity $\left(\mathrm{W} / \mathrm{m}^{\circ} \mathrm{C}\right)$ & 11.35 & 18.13 & -37.34 \\
\hline Thermal resistivity $\left(\mathrm{m}^{\circ} \mathrm{C} / \mathrm{W}\right)$ & 0.03 & 0.01 & 0.69 \\
\hline Thermal diffusivity $\left(\mathrm{mm}^{2} / \mathrm{sec}\right)$ & -0.55 & -0.45 & 20.17 \\
\hline Angle of repose $\left(^{\circ}\right)$ & $\ldots$ & -0.02 & 0.50 \\
\hline Aerated bulk density $\left(\mathrm{kg} / \mathrm{m}^{3}\right)$ & -1.38 & -1.03 & -14.47 \\
\hline Particle size $\left(d_{\mathrm{gw}}, \mathrm{mm}\right)$ & -0.38 & -0.01 & -4.94 \\
\hline Hausner ratio $(-)$ & 2.31 & -0.41 & -4.71 \\
\hline Total flow index (-) & -0.02 & -0.02 & $\ldots$ \\
\hline Jenike flow index $(-)$ & 0.19 & $\ldots$ & $\ldots$ \\
\hline Water activity $(-)$ & -1.40 & -0.77 & -12.59 \\
\hline Protein dispersibility index $(\%)$ & 0.07 & 0.01 & 1.56 \\
\hline Porosity $(\%)$ & 0.08 & -0.48 & 4.26 \\
\hline Compressibility (\%) & -0.05 & 0.01 & 0.14 \\
\hline Angle of spatula $\left({ }^{\circ}\right)$ & -0.004 & -0.11 & 0.42 \\
\hline Uniformity (-) & -0.72 & 0.38 & -1.97 \\
\hline Angle of fall $\left(^{\circ}\right)$ & 0.89 & 0.09 & -0.47 \\
\hline Angle of difference $\left(^{\circ}\right)$ & 0.92 & -0.06 & 0.48 \\
\hline Dispersibility (\%) & 0.03 & -0.01 & 0.23 \\
\hline Total flood index (-) & -0.02 & 0.0006 & 0.10 \\
\hline Total flow index/total flood index (-) & -0.66 & 0.07 & $\ldots$ \\
\hline Total flow index/Jenike flow index (-) & 0.005 & $\ldots$ & $\cdots$ \\
\hline$F$ statistic & 231.17 & $1,121.07$ & 66.14 \\
\hline$P$ value & 0.0001 & 0.0001 & 0.0001 \\
\hline$R^{2}$ & 0.89 & 0.94 & 0.80 \\
\hline PLS components required & 9 & 2 & 10 \\
\hline
\end{tabular}


the data for outliers, reducing the number of variables, without any loss of information, and PCA can be further used for CA programs (Sharma 1996; Johnson 1998).

PLS regression focuses on maximizing the variance of the response as explained by the predictor variables, instead of just producing the empirical covariance matrix. PLS regression could also be used for cases in which sample size is not sufficient, whereas multiple regression methodology needs a sufficiently large dataset (Haenlein and Kaplan 2004). PLS regression is a technique that generalizes and combines the features of PCA and the multiple regression procedure. We had 22 dependent variables and three independent variables for this experimental design. When the number of dependent variables is large compared with independent variables (as in this case), and when the number of predictors (dependent variables) is more than the number of observations, then the multiple regression approach is no longer feasible because of multicollinearity. PCA and PLS regression are some of the approaches used to handle this problem (Abdi 2003). In PCA, the predictor matrix is used to find the required number of principal components, and these principal components are used for further data screening, clustering, and regression modeling. However, in PLS modeling, a set of latent vectors are formed from the predictor matrix; the latent vectors perform simultaneous decomposition of predictors and response variables, finally yielding a regression equation for the response variables (Abdi 2003).

As shown in Table $\mathrm{V}$, out of all dependent variables, only AoR $\left(^{\circ}\right)$, Jenike flow index $(-)$, and total flow index/Jenike flow index (-) yielded a PLS regression equation with $R^{2} \geq 0.80$. In this study, we tried to perform PLS regression with previously determined dimensionless flow parameters (such as total flow index/total flood index [-] and HR [-] from Bhadra et al [2009c]), but we could not find many results with $R^{2} \geq 0.80$. Thus, in this study we did not include all results and only depicted results for AoR $\left(^{\circ}\right)$, Jenike flow index (-), and total flow index/Jenike flow index (-). The highest $R^{2}(0.94)$ was found for Jenike flow index (-), followed by AoR (0.89) and finally total flow index/Jenike flow index (0.80). For Jenike flow index (-), the optimum number of principal components was found to be 2 . However, for AoR $\left(^{\circ}\right)$ and total flow index/Jenike flow index $(-)$, the numbers of principal components were 9 and 10, respectively. The $F$ value was highest for Jenike flow index $(1,121.07)$, followed by AoR (231.17) and then total flow index/Jenike flow index (66.14). The parameter estimates for each response variable (AoR $\left[{ }^{\circ}\right]$, Jenike flow index [-], and total flow index/Jenike flow index $[-])$, are listed in Table V.

Figure 8 represents the model selection plot and loading plots for the response variables listed in Table V. As shown in Figure $8 \mathrm{~A}$, the model selection plots were in agreement with the PLS regression output (Table $\mathrm{V}$ ). The best cross-validation results were seen for the Jenike flow index (-), because of the high $R^{2}$ value for the regression model with the minimum number of principal components. Cross-validation is a method to test the fitness of the PLS regression model; in this method, the $i$ th observation is deleted and the model is built up from the remaining observations (Srivastava 2002).

As shown in Figure 8B, for predicting AoR, the compressibility, PDI, and uniformity variables were the most influential variables. For predicting Jenike flow index (-), the CDS level, porosity, and AoS were the most influential variables. However, CDS level, total flood index, dispersibility, porosity, ABD, and total flow index/total flood index were the most influential parameters for predicting total flow index/Jenike flow index (-). The plot that describes the original and new variables is called the loadings, and it indicates to what extent the original variables are influential in forming the new set of variables, for any given response variable. The greater the loading value of the variable is, the higher is the eigenvalue, and thus the loading values contribute more in forming the principal component scores (Johnson 1998).

From PCA based on the covariance matrix, just two principal components were able to represent $96 \%$ of the variability in the total data set without loss of substantial data information. The highest eigenvalue was found for the first principal component. The maximum difference in the eigenvalue comes after two components, as shown in Figure 9A (scree plot), indicating that the critical number of principal components required to repre-
$\operatorname{AoR}\left({ }^{\circ}\right)$

A

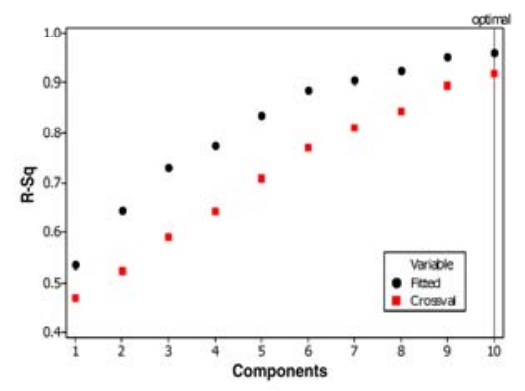

B

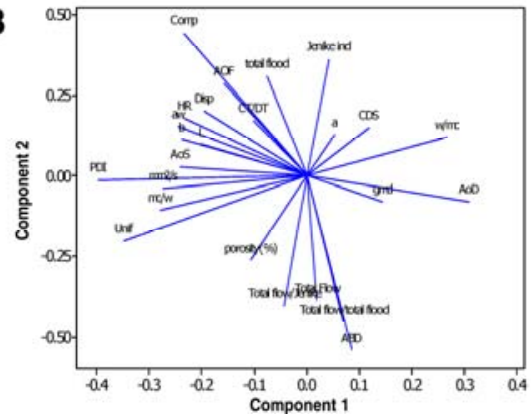

Jenike Flow Index (-)
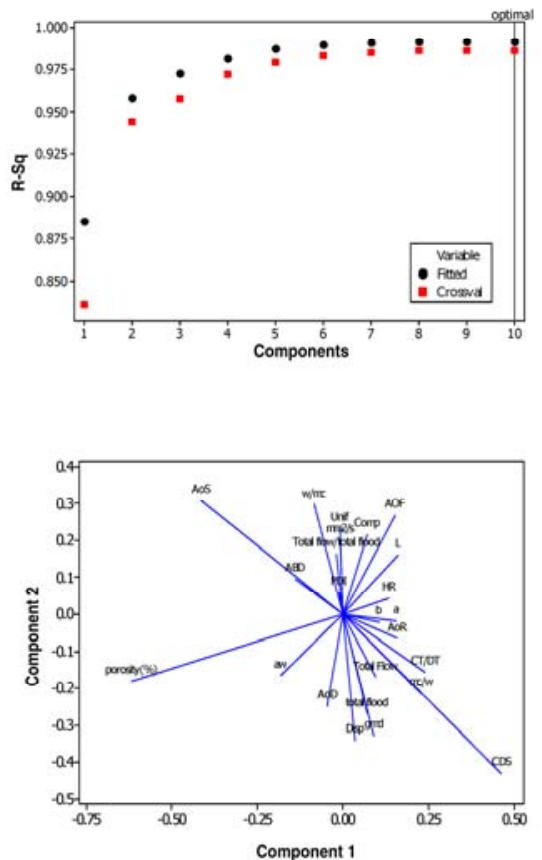

Total Flow Index / Jenike Flow Index (-)
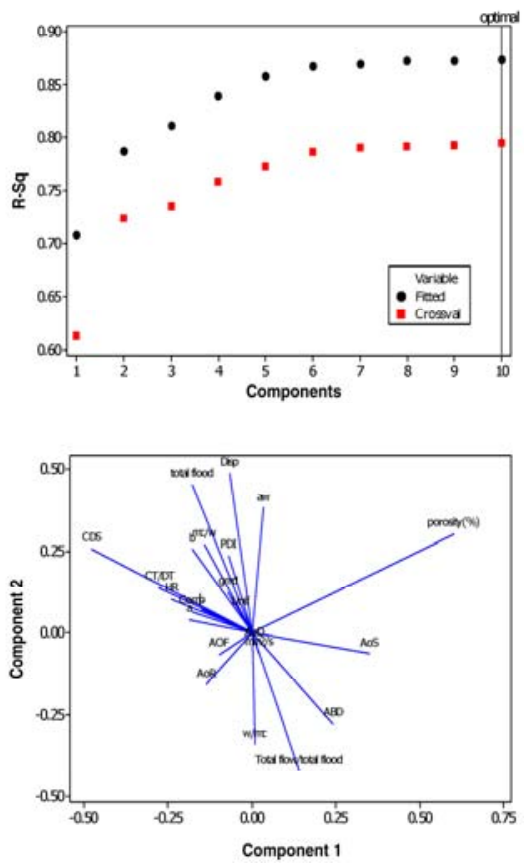

Fig. 8. PLS regression results. A, Model selection plots with cross-validation for angle of repose (AoR, ${ }^{\circ}$ ), Jenike flow index (-), and total flow index/ Jenike flow index ratio $(-)$; B, loading plots for response variables as a function of all other flow and physical properties. 
sent the maximum variability in the dataset was two. Some plots suggest the actual dimensionality of the space in which the whole data set will lie (Johnson 1998). After determining the critical number of principal components, we proceeded toward loading plots for the whole dataset with two principal components only. Figure 9B represents the loading plot for two principal components, and it indicates that cooling temperature and drying temperature were the most influencing factors for the overall flowability study. CDS addition levels were not at all significant in overall flowability properties, agreeing with our previous research on desorption modeling in DDGS samples, which concluded that drying temperature was the main effect and CDS was a subeffect on moisture ratio (Bhadra et al 2009b). After cooling and drying temperatures, total flow index/Jenike flow index (-) was the most influencing parameter for overall flowability (Fig. 9B). Based on the results of the loading plots, we proceeded further to observe the score matrix plot for the complete dataset with two principal components. As shown in Figure 9C (score matrix plot), sharp distinction among the data based on three drying temperatures $\left(100,200\right.$, and $\left.300^{\circ} \mathrm{C}\right)$ was observed. However, we could not find such clear groupings with other independent variables. In PCA, the principal component scores were highest for drying temperature (0.99) and cooling temperature (0.94), which was in agreement with the result of the loading plot (Fig. 8B).

Furthermore, we performed a factor analysis (Johnson 1998) procedure for this dataset, and we also found that two factors were optimum to represent $99 \%$ of the variability in the complete data. For the factor analysis procedure, the highest score corresponded to drying temperature $(-0.87)$, followed by AoR $(-0.74)$ and PDI (0.84). The final communality test with the MINEIGEN criterion did not show any variable with an estimate of $<0.3$, indicating that we cannot remove any dependent variable. Hence, all the abovementioned dependent variables were equally important for flowability analyses. We also performed CA (Johnson 1998) on the data set. Furthermore, a hierarchical tree plot and a scatter plot of CA (Johnson 1998) revealed that our dataset could be divided into three clusters, but there was substantial overlap in the observations, and hence, we were unable to differentiate the groups.

Perhaps one of the reasons that this study did not provide multiple regressions with higher $R^{2}$ values was because there were correlations among the response variables. Such correlation in the response variables and their statistical dependence is taken into account in multivariate statistical tools like PLS, PCA, factor analysis, and CA. Response variables, or dependent variables, are often described as random variables, and their dependence is one of the things to be accounted for in the multivariate analyses. Hence, these response variables are often described by their joint probability distribution. This consideration makes multivariate modeling relatively manageable and provides a convenient framework for scientific analysis of the data.

\section{CONCLUSIONS}

Statistical analyses revealed that for some key physical and flow properties, such as $\mathrm{ABD}$, particle size, and porosity, the cooling temperature variable did not show any significant differences among its levels $\left(-12,25\right.$, and $\left.35^{\circ} \mathrm{C}\right)$. There were significant interaction effects for flowability properties between cooling temperature, CDS level, and drying temperature. Key flow parameters such as HR, AoR, Jenike flow index, ABD, and total flow index showed that there was lower variation among the three cooling temperature levels. Response surface plots yielded the highest $R^{2}$ value of 0.70 for Hunter $L(-)=f$ (cooling temperature/drying temperature [-] and CDS [\%, wb]). However, other important flow parameters such as AoR, Jenike flow index, and HR only yielded an $R^{2}$ range from 0.56 to 0.65 . Multi- variate statistical tools showed more promising results, because of the ability to handle multicollinearity in the dataset. PLS regression modeling yielded a higher $R^{2}$ for AoR (0.89), Jenike flow index (0.94), and total flow index/Jenike flow index (0.80). PCA showed that two principal components were enough to describe $96 \%$ of the entire dataset. Drying temperature and cooling temperature were the most influencing variables for the complete dataset. This study also showed that Jenike flow index (-) was an important parameter for estimating flow characteristics in DDGS with varying drying temperature, cooling temperature, and CDS levels. Future studies with wider temperature and CDS ranges should be investigated to gain a better understanding of flowability in DDGS. Additional studies to examine the effect of storage time can also be an interesting area for further investigation.
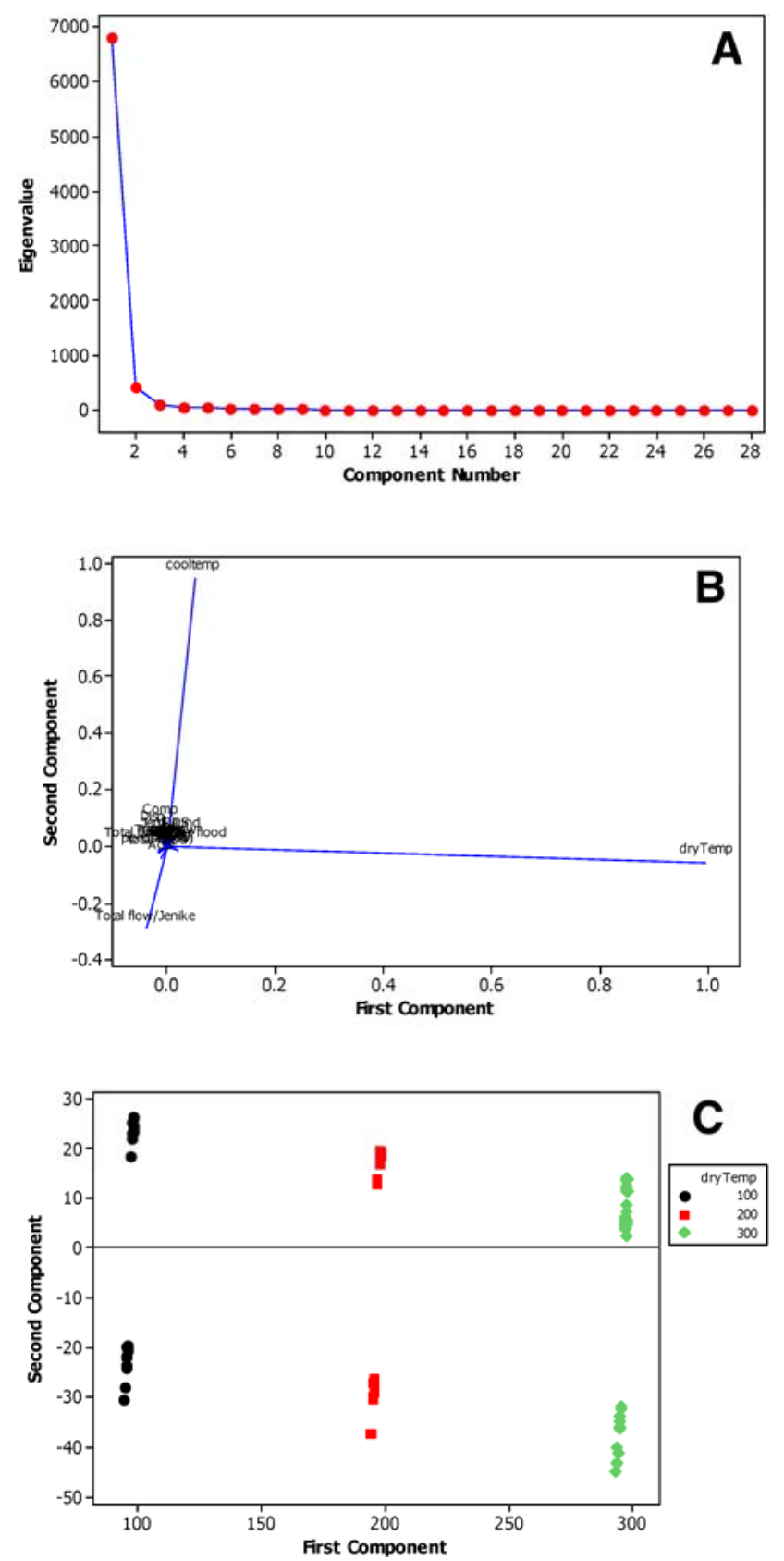

Fig. 9. Principal components results. A, Scree plot used to determine that two principal components were required to summarize $96 \%$ of variability in the data; B, loading vector plot indicates the two most influencing independent variables; $\mathbf{C}$, score plot of the first two principal components indicates classification based on drying temperatures. 


\section{ACKNOWLEDGMENTS}

The authors extend gratitude to Dakota Ethanol, LLC (Wentworth, SD, U.S.A.), who contributed coproduct samples for this study, and to the South Dakota Corn Utilization Council (SDCUC), South Dakota Agricultural Experimental Station, and USDA-ARS for providing facilities, equipment, and financial support.

\section{LITERATURE CITED}

AACC International. Approved Methods of Analysis, 11th Ed. Method 46-24.01. Protein dispersibility index. Approved April 25, 1965. AACC International: St. Paul, MN. http://dx.doi.org/10.1094/AACCIntMethod46-24.01

Abdi, H. 2003. Partial least squares (PLS) regression. In: Encyclopedia of Social Sciences Research Methods. M. Lewis-Beck, A. Bryman, and T. Futing, eds. Sage: Thousand Oaks, CA.

Abu-hardan, M., and Hill, S. 2010. Handling properties of cereal materials in the presence of moisture and oil. Powder Technol. 198(1):16-24.

American Society for Testing and Materials (ASTM). 1999. Method D6393. Standard test method for bulk solids characterization by Carr indices. ASTM: West Conshohocken, PA.

Aulton, M. E., ed. 2001. Pharmaceutics: The Science of Dosage Form Design, 2nd Ed. Churchill: Livingstone, U.K.

Barbosa-Canovas, G., and Juliano, P. 2005. Compression and compaction characteristics of selected food powders. Adv. Food Nutr. Res. 49(1): 233-300.

Bhadra, R., Muthukumarappan, K., and Rosentrater, K. A. 2009a. Flowability properties of commercial distillers dried grains with solubles (DDGS). Cereal Chem. 86(2):170-180.

Bhadra, R., Muthukumarappan, K., Rosentrater, K. A., and Kannadhason, S. 2009b. Desorption studies in DDGS under varying CDS and temperature levels. ASABE International Conference, June 21-24, Reno, NV. Paper No. 095843. ASABE: St. Joseph, MI.

Bhadra, R., Rosentrater, K. A., and Muthukumarappan, K. 2009c. Effects of CDS and drying temperatures levels on flowability behavior of DDGS. ASABE International Conference, June 21-24, Reno, NV. Paper No. 095844. ASABE: St. Joseph, MI.

Bhadra, R., Rosentrater, K. A., and Muthukumarappan, K. 2009d. Measurement and comparison of glass transition and sticky point temperatures for DDGS as affected by CDS and drying temperature levels. ASABE International Conference, June 21-24, Reno, NV. Paper No. 096614. ASABE: St. Joseph, MI.

Bhadra, R., Rosentrater, K. A., and Muthukumarappan, K. 2010. Effects of varying CDS, drying temperature, and cooling temperature levels on glass transition temperatures for DDGS. ASABE International Conference, June 20-23, Pittsburgh, PA. Paper No. 1008603. ASABE: St. Joseph, MI.

Buchheit, J. K. 2002. Distillers dried grains with solubles. Rural Enterprise and Alternative Agricultural Development Initiative Report No. 11. Southern Illinois University: Carbondale, IL.

Carr, R. L., Jr. 1965. Evaluating flow properties of solids. Chem. Eng. 72(3): $163-168$.

Chang, C. S. 1988. Measuring density and porosity of grain kernels using a gas pycnometer. Cereal Chem. 65(1):13-15.

Chegini, G. R., and Ghobadian, B. 2007. Spray dryer parameters for fruit juice drying. World J. Agric. Sci. 3(2):230-236.

Chell, M. 1992. Properties and food applications of soy flours. Pages 306-313 in Proceedings of World Conference on Oilseed Technology Utilization, September 13-18. T. H. Applewhite, ed. Am. Oil Chemists Soc.: Urbana, IL.

Cromwell, G. L., Herkelman, K. L., and Stahly, T. S. 1993. Physical, chemical, and nutritional characteristics of distillers dried grains with solubles for chicks and pigs. J. Anim. Sci. 71(3):679-686.

Cruz, C. R., Brouk, M. J., and Schingoethe, D. J. 2005. Lactational response of cows fed condensed corn distillers solubles. J. Dairy Sci. 88:4000-4006.
Fitzpatrick, J. J., Iqbal, T., Delaney, C., Twomey, T., and Keogh, M. K. 2004. Effect of powder properties and storage conditions on the flowability of milk powders with fat contents. J. Food Eng. 64(4):435-444.

Ganesan V., Rosentrater, K. A., and Muthukumarappan, K. 2007a. Modeling the flow properties of DDGS. Cereal Chem. 84(6):556-562.

Ganesan, V., Rosentrater, K. A., and Muthukumarappan, K. 2007b. Physical and flow properties of regular and reduced fat distillers dried grains with solubles (DDGS). Food Bioprocess Technol. 2(2):156-166.

Ganesan, V., Muthukumarappan, K., and Rosentrater, K. A. 2008. Flow properties of DDGS with varying soluble and moisture content using Jenike shear testing. Powder Technol. 187(2):130-137.

Geldart, D., Abdullah, E. C., and Verlinden, A. 2009. Characterization of dry powders. Powder Technol. 190(1):70-74.

Grey, R. O., and Beddow, J. K. 1969. On the Hausner ratio and its relationship to some properties of metal powders. Powder Technol. 2(6):323-326.

Haenlein, M., and Kaplan, A. M. 2004. A beginner's guide to partial least squares analysis. Understanding Stat. 3(4):283-297.

Hunter Associates Laboratory. 2002. Universal Software User's Manual, Version 2.5. Hunter Associates Laboratory: Reston, VA.

Jenike, A. W. 1964. Storage and flow of solids. Bulletin No. 123. Utah Engineering Station, University of Utah: Salt Lake City, UT.

Johanson, J. R. 1972. Modeling flow of bulk solids. Powder Technol. 5(2):93-99.

Johnson, D. E. 1998. Applied Multivariate Methods for Data Analysis, 1st Ed. Brooks/Cole: Pacific Grove, CA.

Kamath, S., Puri, V. M., and Manbeck, H. B. 1994. Flow property measurement using the Jenike cell for wheat flour at various moisture contents and consolidation times [Letter]. Powder Technol. 81(2):293-297.

Kleinschmit, D. H., Schingoethe, D. J., Kalscheur, K. F., and Hippen, A. R. 2006. Evaluation of various sources of corn distillers dried grain plus solubles (DDGS) for lactating dairy cattle. J. Dairy Sci. 89(12):4784-4794.

Knowlton, T. M., Carson, J. W., Klinzing, G. E., and Yang, W. C. 1994. The importance of storage, transfer, and collection. Chem. Eng. Prog. 90(1):44-54.

Qin, G. X., Verstegen, M. W. A., and van der Poel, A. F. B. 1998. Effect of temperature and time during steam treatment on the protein quality of full fat soybean from different origins. J. Sci. Food Agric. 77(3): 393-398.

Renewable Fuels Association. 2012. Industry resources: Coproducts. Available at: www.ethanolrfa.org/pages/industry-resources-coproducts

Rock, M., and Schwedes, J. 2005. Investigations on the caking behavior of bulk solids-Macroscale experiments. Powder Technol. 157(1-3): 121-127.

Rosentrater, K. A., and Muthukumarappan, K. 2006. Corn ethanol coproducts: Generation, properties, and future prospects. Int. Sugar J. 108(1295):648-657.

Sahin, S., and Sumnu, S. G. 2006. Physical Properties of Foods, 1st Ed. Wiley: New York, NY.

Sharma, S. 1996. Applied Multivariate Techniques, 1st Ed. Wiley: New York, NY.

Speigel, M. R. 1994. Statistics, 3rd Ed. McGraw Hill: New York, NY.

Speihs, M. J., Whitney, M. H., and Shurson, G. C. 2002. Nutrient database for distiller's dried grains with solubles produced from new ethanol plants in Minnesota and South Dakota. J. Anim. Sci. 80(10): 26392645.

Srivastava, M. S. 2002. Methods of Multivariate Statistics, 1st Ed. Wiley: New York, NY.

Teunou, E., and Fitzpatrick, J. J. 2000. Effect of storage time and consolidation on food powder flowability. J. Food Eng. 43(1):97-101.

Thomas, J., and Schubert, H. 1979. Particle characterization. Pages 301319 in: Proceedings of Partec 79. Nuremberg, Germany.

Thomas, M., van Kol Edwin, M. R. A. H., Tamminga, S., Verstegen, M. W. A., and van der Poel, A. F. B. 1997. Effect of water, steam and shear conditioning on the protein quality of soy grits. J. Sci. Food Agric. 74(3):392-400.

[Received April 10, 2012. Accepted October 25, 2012.] 